\title{
ORIENTATION CHARACTERISTICS OF THE MICROSTRUCTURE OF HIGHLY ROLLED PURE COPPER AND PHOSPHORUS-COPPER
}

\author{
K. SZTWIERTNIA and F. HAESSNER \\ Institut für Werkstoffe der Technischen Universität Braunschweig, Langer Kamp \\ 8, D-W-3300 Braunschweig, Germany
}

\begin{abstract}
Orientational aspects of the mutual arrangement of crystallites in a microstructure can be described by certain distribution functions of orientation or orientation differences. Among these functions the "principle" distributions are completely determined by the texture, whereas the "partial" distributions can be used to describe correlations between orientations. The most interesting partial distribution, the misorientation distribution function (MODF), concerns nearest neighbourhood relations. The basic source of information about all of these descriptions is the spatial arrangement of orientations in the sample. In the present case the local orientation arrangement in $95 \%$ rolled pure copper and phosphorus copper was determined in the TEM using Kikuchi-patterns.

The analysis of the data show that:

(i) the orientational details vary in a smaller lateral scale in phosphorus copper than in pure copper.

(ii) the spatial arrangement of orientations in the sample is by no means random: spatial orientation "clustering" or "ordering" may occur.

(iii) the MODF, which shows the distribution of orientation differences between neighboured areas in the microstructure, clearly reveals in both materials a strong preference for $\sim 60^{\circ}\langle 111\rangle$ and $\sim 50^{\circ}\langle 110\rangle$ relations. These and practically all other orientation differences found in the materials correspond to the Coincidence Site-Lattice (CSL) orientation relationships.
\end{abstract}

KEY WORDS Microstructure, orientation distributions, misorientation distributions

\section{INTRODUCTION}

Many properties of polycrystalline materials and processes in the solid state are influenced by the mutual arrangement of individual crystallites, that is the "orientation topography" (see: Haeßner et al. (1983)). A review of the orientation distribution functions, which can be used to describe certain characteristics of this type of orientational map, may be found in a monograph by Bunge (1982). A very general characterization of the orientation relationships in a microstructure is the conditional form of the two-point orientation correlation function COCF $c\left(g^{\prime}, g^{\prime \prime} \| \mathbf{r}\right)$ discussed by Adams et al. (1987). This function gives the probability density of the occurrence of orientations $g^{\prime}$ and $g^{\prime \prime}$ when they are separated by a fixed vector $\mathbf{r}$. Of special importance in this kind of analysis are one-point forms of distribution which can be represented in 3-dimensional orientation space. It can be shown that these microstructural orientation characteristics can be derived in most cases from the COCF (Morawiec and 
Pospiech, 1991), or directly defined on the set of measured orientations (Pospiech et al., 1991). The basic source of information about all of these descriptions is the spatial arrangement of orientations in the sample.

In this paper an example of this structural analysis of orientation maps of $95 \%$ rolled pure copper and phosphorus-copper is presented. The local orientation distribution was determined in the TEM using Kikuchi-patterns.

\section{ORIENTATION CHARACTERISTICS}

In the following all distribution functions characterizing the orientation aspects of the microstructure, i.e. which can be represented in orientation space, will be denoted orientation characteristics. The characteristics which we use in this paper, are described and classified in detail by Pospiech et al., (1991). We will briefly list their most important features.

Among the different functions one can distinguish between "principal" and "partial" distributions. "Principal distributions" are completely determined by the texture, i.e. they are formed by the whole set of measurements irrespective of the locations of the measuring points in the sample. "Partial distributions" encompass only subsets of the whole group of orientation data.

The first of the principal distributions is the well-known Orientation Distribution Function ("ODF") $f(g)$ which describes the texture, i.e. the frequency of orientations $g$ in the material and which is the most important characteristic of the microstructure. It is defined by the expression:

$$
\Delta N(g) / N=f(g) \Delta g
$$

where $N$ is the total number of the measuring points in the sample space and $\Delta N / N$ is the frequency of occurrence of an orientation in the range $\Delta g$ around $g$.

The second one is the Orientation Difference Distribution Function ("ODDF") $u(\Gamma)$ which describes the frequency of the differences $\Gamma$ between orientations $g$ and $g^{\prime}$ occurring for any two measuring points $P, P^{\prime}$ in the sample:

$$
\Delta v(\Gamma) / v=u(\Gamma) \Delta \Gamma
$$

where $v$ is the total number of pairs of measuring points and $\Delta v / v$ is the frequency of occurrence of the orientation difference inside the range $\Delta \Gamma$ around $\Gamma$ irrespective of the position of the chosen two measuring points in the sample space.

By setting $g^{\prime}=\Gamma g$ (i.e. $\left.\Gamma=g^{\prime} g^{-1}\right) u(\Gamma)$ can be derived from $f(g)$ by integrating according to:

$$
u(\Gamma)=\int_{G} f(g) f(\Gamma g) d g
$$

where $G$ is the total orientation space.

The functions $f(g)$ and $u(\Gamma)$ represent the particular cases of one-point forms of distributions obtained by integration of the two-point COCF (Adams et al., 1987).

The "partial distributions" may be used for a more detailed investigation of correlations which possibly exist between various orientations in a polycrystalline 
matrix, i.e. for comprehensive specification of the microstructure. Some of them are analytically related to the two point COCF. There exist partial distribution functions of orientations (partial ODFs $H(g)$ ) and of orientation differences (partial ODDF's $V(\Gamma)$ ). Since here only subsets of the measured orientations or orientation differences are used, individual measured values are weighted differently. This is expressed by using weight factors $Q_{i} \leq 1$, or $T_{j} \leq 1$, assigned to the orientation measuring points $P_{i}$, or to pairs $P_{j}$ of measuring points when orientation differences are considered respectively.

Using the weight factors, the partial distributions are defined (and normalized to unity) in the same way as the principal distributions. Thus the partial ODF $H(g)$ is defined by

$$
H(g) \Delta g=\Delta Q(g) / Q=[\Delta Q(g) / \Delta N(g)][\Delta N(g) / N][N / Q]
$$

and

$$
H(g)=[\Delta Q(g) / \Delta N(g)][f(g) / \bar{Q}]=K(g) f(g) / \bar{Q}
$$

where: $Q=\sum Q_{i}$ for $i=1, \ldots, N, \Delta Q(g)=\sum Q_{i}$ for $i \in \Delta g$ around $g, \bar{Q}=Q / N$.

The function $K(g)=\Delta Q(g) / \Delta N(g)$ in Eq. (4) represents an orientation characteristic where the respective statistical weight $Q_{i}$ of single measuring points of the sample space is transformed to orientation space. The product $K(g) f(g)$ in the expression describes the weighted fraction of the ODF density for a given orientation. Analogously, the partial ODF $V(\Gamma)$ is defined by using the weight factors $T_{j}$ to select the orientation differences:

$$
V(\Gamma) \Delta \Gamma=\Delta T(\Gamma) / T=[\Delta T(\Gamma) / \Delta v(\Gamma)][\Delta v(\Gamma) / v][v / T]
$$

and

$$
V(\Gamma)=[\Delta T(\Gamma) / \Delta v(\Gamma)][u(\Gamma) / \bar{T}]
$$

where: $T=\sum T_{j}, \Delta T(\Gamma)=\sum T_{j}$ for $j \in \Delta \Gamma(\Gamma), \bar{T}=T / v$.

Reducing $H(g)$ or $V(\Gamma)$ with respect to the ODF $f(g)$ or to the ODDF $u(\Gamma)$, finally gives:

$$
\begin{gathered}
h(g)=H(g) / f(g)=K(g) / \bar{Q} \\
v(\Gamma)=V(\Gamma) / u(\Gamma)
\end{gathered}
$$

The texture reduced partial distributions $h(g), K(g)$ and $v(\Gamma)$ are called "preference functions". They express how much more frequently an orientation or orientation difference occurs when weight factors are applied, compared to the case without.

An important special case for $h_{g_{0} r}(g)$ is given by those orientations whose measuring points $P^{\prime}$ are separated from the locations $P_{0}$ of a special orientation $g_{0}$ by the vector $\mathbf{r}$, or by the distance $|\mathbf{r}|$, i.e. $Q_{i}=1$, for points $P_{i}^{\prime}$ and $Q_{i}=0$, for all other points. For a sufficiently large distance $|\mathbf{r}|$ from $P_{0}$ the correlations disappear and one obtains $h_{g_{0} r}(g) \cong 1$.

The most essential form of the preference ODDF $v(\Gamma)$ is obtained by selecting pairs of such points which are separated by the fixed vector $\mathbf{r}$, or by the distance $|\mathbf{r}|$. If the distance $|\mathbf{r}|$ is sufficiently large, then the correlations disappear leading to $v(\Gamma) \cong 1$. Of great importance is the other boundary case in which $|\mathbf{r}|$ is the nearest neighbour distance. The resulting partial ODDF, the misorientation distribution function MODF $V(\Gamma) \equiv M(\Gamma)$, describes the density distribution of 
orientation differences $\Gamma$ to the nearest neighbour measuring points (Pospiech et $a l ., 1986)$. Its texture reduced form, the preference $\operatorname{MODF} m(\Gamma)=M(\Gamma) / u(\Gamma)$, shows which misorientations occur more frequently as are expected from texture calculations. A further kind of partial ODDF is defined on the subset of orientation differences $\Gamma$ selected by additional conditions. An important example is based on the condition that the pairs of orientations separated by the fixed distance $|\mathbf{r}|$ contain the special orientation $g_{0}$. In order to obtain the preference function $v_{g_{0} r}(\Gamma)$ it appears reasonable to relate $V_{g_{0} r}(\Gamma)$ not to the ODDF $u(\Gamma)$, but to the MODF $M(\Gamma)$ :

$$
v_{g_{0} r}(\Gamma)=V_{g_{0} r}(\Gamma) / M(\Gamma)
$$

In the following we call all above defined functions $s(t)$, where $t$ means orientations or orientation differences.

\section{DETAILS OF THE EXPERIMENT AND CALCULATIONS}

Through special thermo-mechanical treatment soft samples of pure copper (purity $99.9998 \%)$ and phosphorus copper $(\mathrm{Cu}+0.96 \% \mathrm{P})$ were produced which showed a fairly random texture for a mean grain size of $\sim 100 \mu \mathrm{m}$ and $\sim 30 \mu \mathrm{m}$, respectively. The material was $95 \%$ reversibly cold-rolled. The ratio $l_{d} / h\left[l_{d}\right.$ : compressed length, $h$ : sample thickness before each rolling step] varied here between 1 to 5 . In this way homogeneous deformation conditions are ensured throughout the sample cross-section. Using this material, thin foils perpendicular to the normal direction $\dagger$ were prepared. In the TEM, at an acceleration voltage of $120 \mathrm{kV}$, bright field images and Kikuchi-patterns (nanobeam electron diffraction) were obtained. The orientations were calculated off-line from the Kikuchi-patterns (Heilman et al., 1982; Weiland and Schwarzer, 1986). The measuring points were located in a quadratic grid at distances of $1 \mu \mathrm{m}$ (the measuring field was chosen at random). For both materials the following number of single orientations were studied:

$$
\begin{aligned}
& \mathrm{Cu}-606 \text { orientations on } 10 \text { foils } \\
& \mathrm{Cu}+0.96 \% \mathrm{P}-384 \text { orientations on } 6 \text { foils }
\end{aligned}
$$

The accuracy of the orientation determination is about $1^{\circ}$ in the reference system of the microscope. Since the determination of the sample coordinate system is rather more difficult, the orientation specification in the sample system is only accurate to within $\sim 5^{\circ}$.

From the measured individual orientations, distribution functions $s(t)$ of the orientations $t=g$ (ODF) and of the orientation differences $t=\Gamma$ (ODDF) were calculated by the method of Fourier series expansion of generalized spherical harmonics $T_{l}^{m n}$ (Bunge, 1982). In the calculations an expansion range $l_{\max }=34$ was applied and for the ODF a cubic/orthorombic $\ddagger$ and for the ODDF cubic/cubic symmetry was assumed.

\footnotetext{
$\dagger$ We have also analysed foils perpendicular to the transverse direction, there the microstructure shows specific inhomogeneities (for instance shear bands). These results will be treated in a separate paper.

$\ddagger$ Additionally measured (by X-ray) pole figures demonstrated well-defined orthorombic symmetry.
} 
In order to obtain continuous distribution functions from the measured discrete orientations, scattering was introduced around each measured value $t$ in the form of a gaussian frequency distribution with width $\varepsilon$ assumed to be $5^{\circ}$ for ODFs and $3^{\circ}$ for ODDFs. This leads to the expression (Pospiech and Lücke, 1975):

$$
s(t)=(1 / M) \sum_{l, m, n} R(\varepsilon) T_{l}^{m n}(t) \sum_{i} W_{i} T_{l}^{* m n}\left(t_{i}\right)
$$

where: $R(\varepsilon)=\left(\exp \left(-l^{2} \varepsilon^{2} / 4\right)-\exp \left(-(l+1)^{2} \varepsilon^{2} / 4\right) /\left(1-\exp \left(1-\varepsilon^{2} / 4\right)\right), \quad M\right.$ is the total number of points $P_{i}(M=N)$, or of pairs of points $P_{j}(M=v)$, and $W_{i}$ a weight factor which is assigned to the various points (Eq. (4)) or pairs (Eq. (5)) in the sample.

Distributions of orientations (ODFs and partial ODFs) are then plotted here in Euler angles space $\left(\phi_{1}, \Phi, \phi_{2}\right)$ in the range $0^{\circ}$ to $90^{\circ}$. By contrast, distributions of orientation differences (ODDFs and partial ODDFs), are recalculated and plotted in the space formed by axis of rotation and rotation angle coordinates $(a, \omega)$, most convenient here for description and interpretation (Pospiech et al., 1986). In a first step the ODF $s(t)=f(g)$ and the ODDF $s(t)=u(\Gamma)$ were calculated writing all $W_{i}=1$ in Eq. (9) for these cases. In order to characterize the orientation correlation to the nearest neighbourhood around the measured points in the grid of the sample space, two kinds of preference ODFs $s(t)=h(g), K(g)$ and two kinds of preference ODDFs $s=v(\Gamma), m(\Gamma)$ were evaluated.

\section{RESULTS}

\section{The Principal Orientation Characteristics}

The ODF of pure copper is shown in Figure 1a. This distribution exhibits the typical rolling texture components of pure fcc metals with high stacking fault energy which take the $B=\{110\}\langle 112\rangle, C=\{112\}\langle 111\rangle$ and $S=\{123\}\langle 364\rangle$ orientations $(\sim 9 \%(12 \%), \sim 18 \%(20 \%)$ and $\sim 38 \%(44 \%)$ of measured points, respectively), $\dagger$ but its general appearance differs somewhat from that most frequently observed in highly rolled copper (Pospiech and Lücke, 1975; Virnich, 1979; Haessner et al., 1983). The distribution is dominated by orientation concentrations in the vicinity of the position $S$, whereas the densities in the positions $C$ and particularly $B$ are relatively weak.

Also the scattering regions of orientation densities compared to those of typical rolling textures are more extended and some irregularities can be noticed. Thus two orientation tubes can be recognized: the minor one (marked by $Y$ ) is for $\Phi=$ const $=35^{\circ}$ and $\phi_{1}+\phi_{2}=115^{\circ}$, and the major one (marked by $X$ ) for $\Phi=$ const $=35^{\circ}$ and $\phi_{1}+\phi_{2}=125-130^{\circ}$. This last tube can be interpreted as wide scattering around the $S$ position.

The ODF of phosphorus copper is shown in Figure 1b. This distribution exhibits the typical rolling texture components of the brass type (Virnich, 1979) which take the $B=\{110\}\langle 112\rangle, \quad S=\{123\}\langle 634\rangle$ and $G=\{110\}\langle 001\rangle$ orientations $(\sim 21 \%(28 \%), \sim 17 \%(20 \%), \sim 8 \%(13 \%)$ of measured points, respectively). $\dagger$

$\dagger$ All orientations which have a disorientation angle $\omega$ relative to $\{h k l\}\langle u v w\rangle$ smaller than $12^{\circ}$ (or than $15^{\circ}$-values in brackets) are counted under $\{h k l\}\langle u v w\rangle$. If in special cases two kinds of characterization were possible (e.g. $B$ and $S$ ), that which fitted best was chosen. 

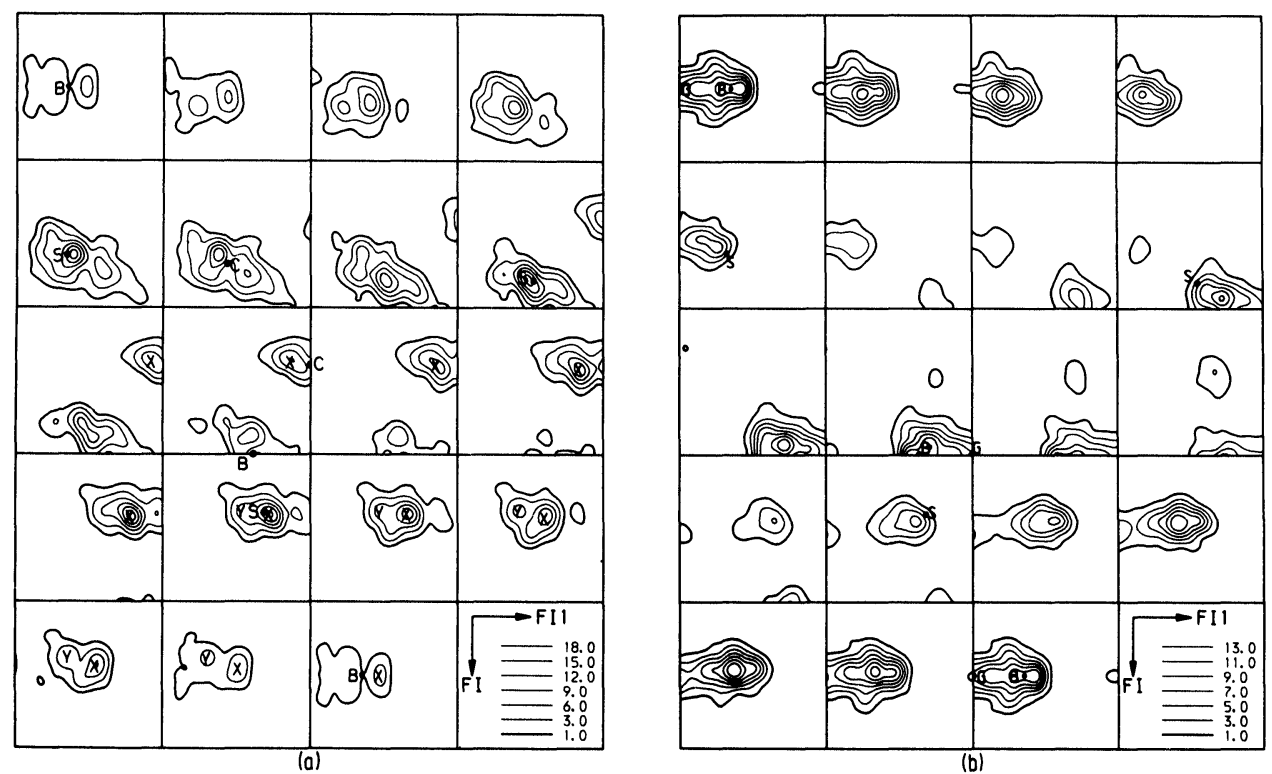

Figure 1 Orientation distribution function ODF calculated from single orientations measured of rolled samples of: a) pure copper, 606 orientations; b) phosphorus copper, 384 orientations. (Intensity levels are listed in the last section plot of the figure).

The ODDFs which, according to Eq. (3), depend only on the ODF, are calculated by using all possible orientation differences in the set of measured orientations. They serve as a reference quantity for partial ODDFs. The ODDF of pure copper is presented in Figure 2a.

In this ODDF a very strong maximum (not shown here) appears near $\omega=0^{\circ}$ with a density $u\left(\omega=0^{\circ}\right)>100$, i.e. many times larger than that for all the other
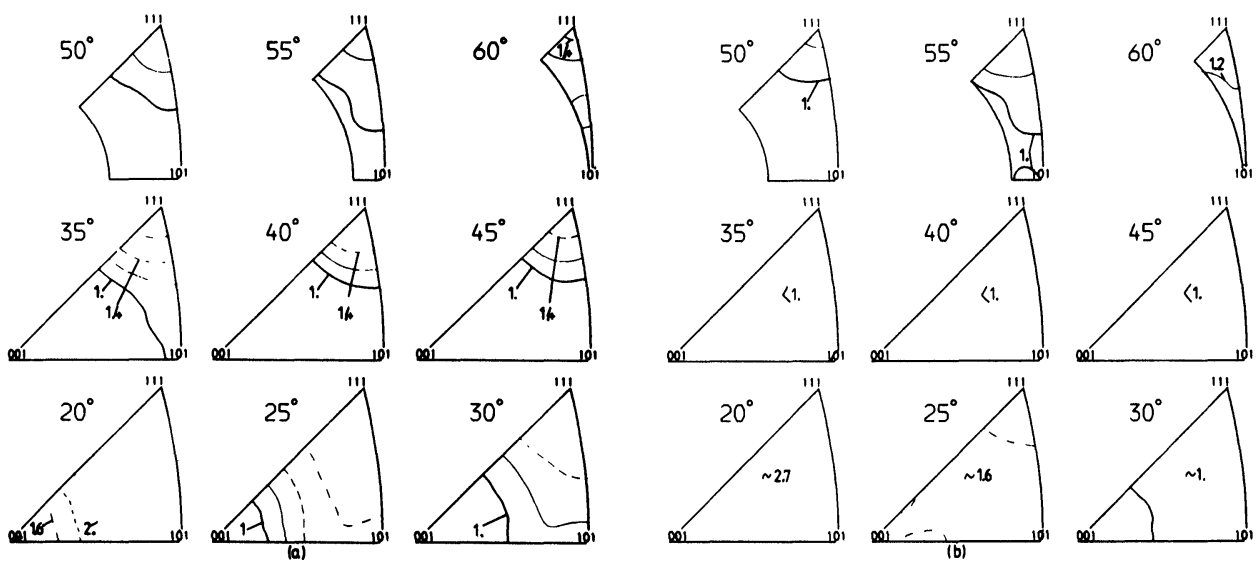

Figure 2 Orientation difference distribution function ODDF determined by texture of rolled samples of: a) pure copper, levels at $1,1.2,1.4, \ldots$, b) phosphorus copper, levels at $1,1.2,1.4, \ldots$ 
maxima. Since the scattering from this high peak dominates large parts of the orientation space and, by its irregularities, severeley falsifies the structure of the density distributions in the lower density regions, in Figure 2 (and in other figures which show ODDFs or partial ODDFs) all orientation differences with $\omega_{j} \leq 12^{\circ}$ have been omitted. This appears reasonable since this peak contains only little relevant information: It stems from orientation pairs for which both orientations lie inside the same peak of the orientation distribution. In addition the axes of rotation for small values of $\omega\left(\omega<10^{\circ}\right)$ show no preferred orientations, i.e. they are more or less randomly distributed.

In Figure $2 \mathrm{a}$ the most probable axes of rotation for $15^{\circ}<\omega<35^{\circ}$ are widely scattered in the band between the $\langle 110\rangle$ and $\langle 111\rangle$ directions, for $35^{\circ}<\omega<60^{\circ}$ they are concentrated around the $\langle 111\rangle$ direction and for $\omega \cong 60^{\circ}$ around the $\langle 221\rangle$ direction as well. Such a distribution corresponds to the orientation differences between the various peaks in the ODF and also to those between the different symmetrically equivalent variants of the ODF. These variants are not visible in the symmetricised ODF but they can be distinguished by means of the calculated individual orientation differences.

The ODDF of phosphorus copper is presented in Figure 2b. This ODDF is much more scattered as in the case of pure copper. This can be related to the relatively high scattering of the orientation distribution (more than $50 \%$ of the orientations have greater distances to $B, S, G$ components than $12^{\circ}$ ). Up to $\omega \cong 30^{\circ}$ the axes of rotation are more or less randomly distributed. For $50^{\circ}<\omega<60^{\circ}$ the axes of rotation are widely scattered around the $\langle 111\rangle$ direction and for $\omega \cong 60^{\circ}$ additionally around the $\langle 110\rangle$ direction.

\section{The Partial Orientation Characteristics}

Partial ODFs selected with respect to the disorientation angles. Here the weight factor $Q_{i}$ for each point $P_{i}$ of the grid characterized by the orientation $g_{i}$ is defined as the fraction $N_{i} / M$, where $N_{i}$ is the number of points $P_{j}$ which lie at a fixed distance $|\mathbf{r}|$ from the point $P_{i}$ and possess a defined orientation relationship with respect to the orientation $g_{i}, M$ is the number of all points $P_{j}$ lying within the distance $|\mathbf{r}|$ from the point $P_{i}$. In the present case two classes of orientations $g_{j}$ have been defined according to their disorientation angles $\omega$, namely $\omega_{a}<8^{\circ}$, and $\omega_{b}>20^{\circ}$. This leads to two sets of weight factors ascribed to the orientation $g_{i}$ : $Q_{i}^{a}=N_{i}\left(\omega_{a}<8^{\circ}\right) / M, Q_{i}^{b}=N_{i}\left(\omega_{b}>20^{\circ}\right) / M$. They give the relative number of crystallites in the distance $|\mathbf{r}|$ from $P_{i}$ whose disorientation angles are not larger than $8^{\circ}$ or are larger than $20^{\circ}$, respectively.

Figures $3 \mathrm{a}, \mathrm{b}$, c, d show the preference ODFs $K(g)=Q \times H(g) / f(g)$ from orientations weighted by the above factors $Q_{i}^{a}$ (i.e. for $\omega<8^{\circ}$ ) and $Q_{i}^{b}$ (i.e. for $\omega>20^{\circ}$ ) and $|\mathbf{r}| \cong 1 \mu \mathrm{m}$ for pure and phosphorus copper respectively. These functions will now be compared to the ODFs $f(g)$ of Figure 1a and b. According to Eq. (4) the partial $H(g)$ can be lower or higher than $f(g)$ : it is $H(g)=0$ for $f(g)=0$ or if all weight factors assigned to the orientation $g$ in the sample space are equal to zero, i.e. $\Delta Q(g)=0$. It is $H(g) \approx f(g)$ when the mean weight factor $K(g)$ assigned to the orientation $g$ is near that of the mean weight factor $\bar{Q}$ taken from all measuring points i.e. $K(g) \cong \bar{Q}$. Thus the preference function $K(g)$ 


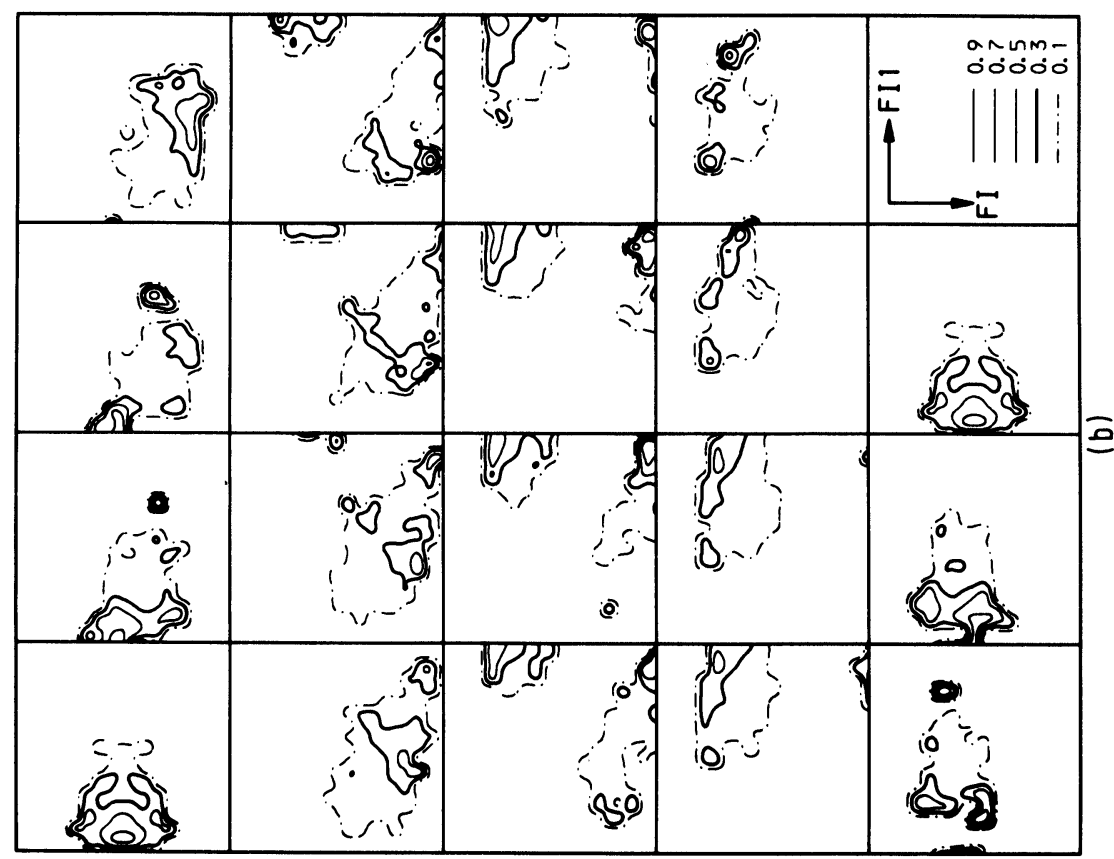

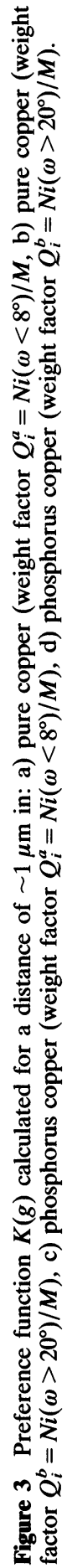

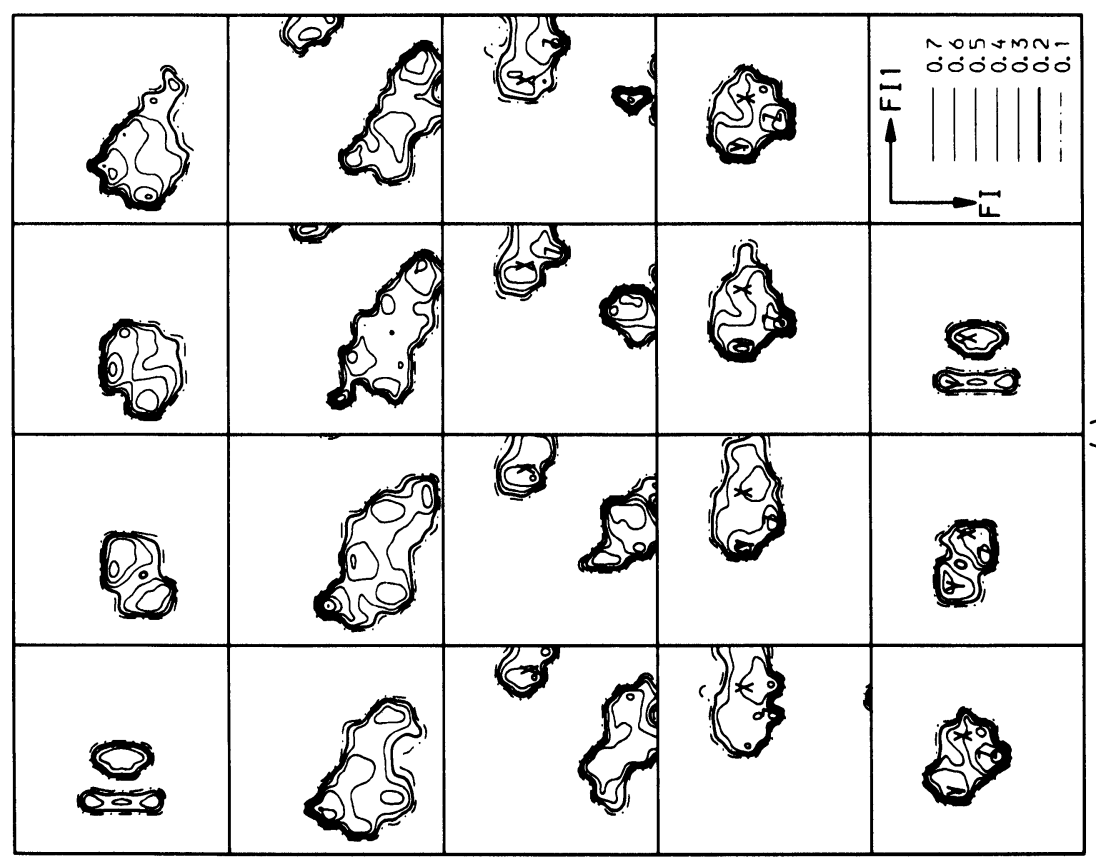


ORIENTATION CHARACTERISTICS

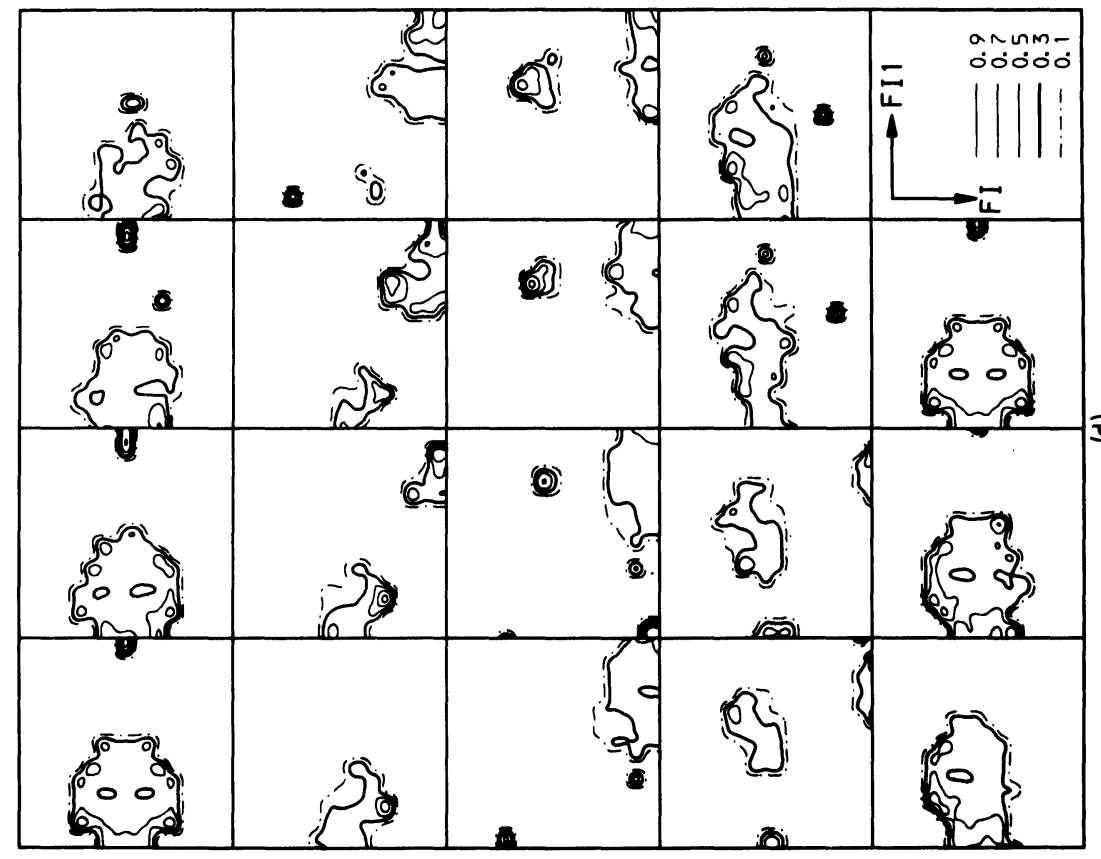

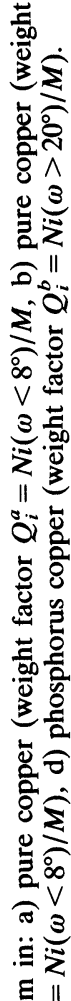

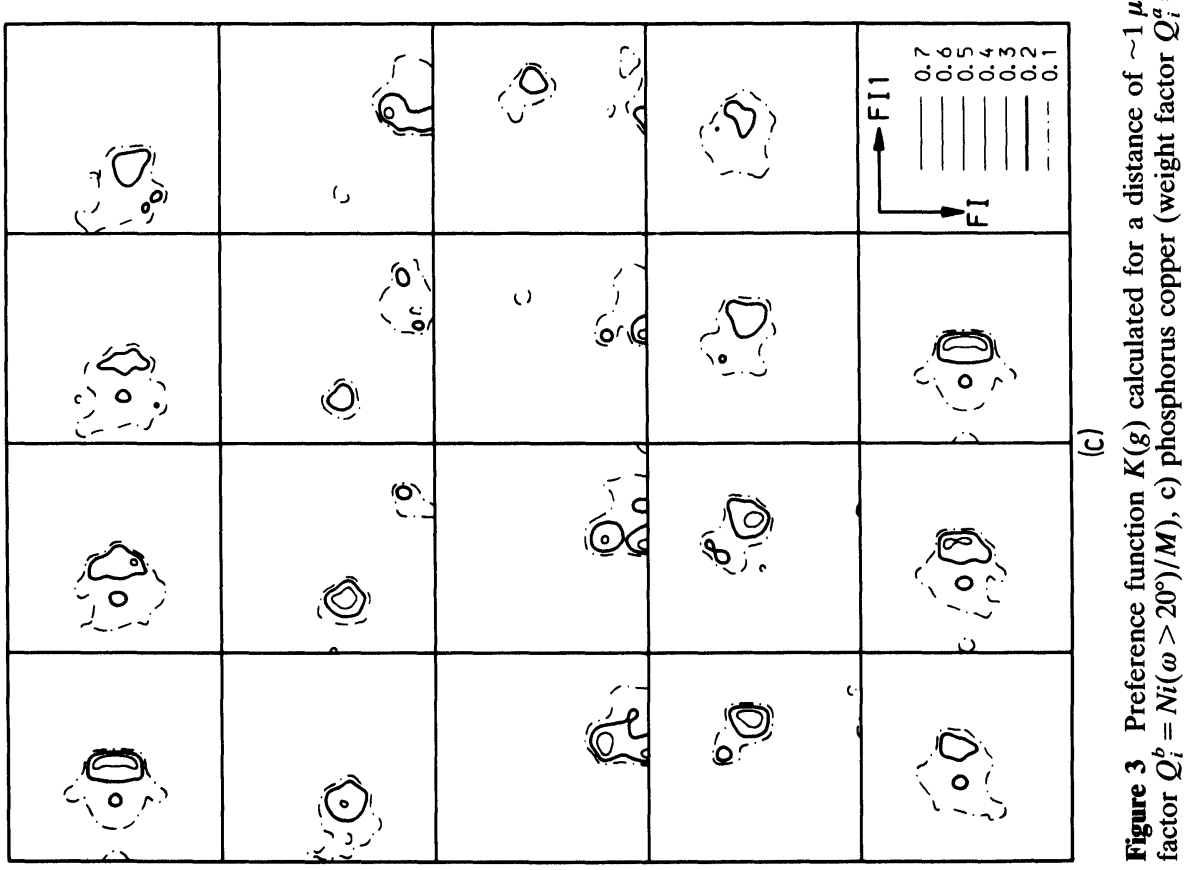


shows directly which contribution in the neighbourhood of a grain with a definite orientation has grains disoriented to its with $\omega<8^{\circ}$ or $\omega>20^{\circ}$.

The comparison of the preference ODF $K_{a}(g)\left(\omega<8^{\circ}\right)$ for pure copper Figure $3 a$ with the ODF in Figure 1a shows considerable differences in the densities; however, the positions of the maxima largely agree. Strongly exposed are the tubes $X$ and $Y$. Quantitatively the contribution of neighbours with $\omega<8^{\circ}$ around an orientation $g_{i}$ out of the tubes $X$ and $Y$ varied between $40 \%$ and $50 \%$. In the $K_{a}(g)$ (Figure 3a) one can identify one further tube (marked by $Z$ ) with $\Phi \cong 45^{\circ}$ and $\phi_{1}+\phi_{2} \cong 125^{\circ}$ for which the mean contribution of neighbours with $\omega<8^{\circ}$ varied between $40 \%$ and $70 \%$. Generally, for orientations out of the tubes $X, Y$ and $Z$ the number of neighbours with small disorientation angles is higher in comparison with those orientations which represent the typical rolling positions. This is shown by the preference function $K_{a}(g): S, C$ and $B$ positions have on the average only about $30 \%$ of neighbours with $\omega<8^{\circ}$.

It cannot be decided whether pairs of measuring points with $\omega<8^{\circ}$ and with $|\mathbf{r}| \cong 1 \mu \mathrm{m}$ are situated within the range of the same grain or in different grains, but the low values of $K_{a}(g)$ indicate that two neighbouring measuring points are rarely situated within the region of the same grain.

Figure $3 \mathrm{~b}$ shows the preference function $K_{b}(g)\left(\omega>20^{\circ}\right)$ for pure copper. In this case one can observe the preference of orientations lying in the scattering around the $C$ position and around the $\sim B$ position. They often form tubes lying parallel to the skeleton line of the main components. Areas with orientations lying in the scattering around the $C$ position have on the average $50 \%$ and those from the scattering around $\sim B 50-70 \%$ of neighbours with $\omega>20^{\circ}$. S-oriented areas have not more than $20 \%$ of neighbours with $\omega>20^{\circ}$.

The same functions as above but calculated for phosphorus copper are shown in the Figures $3 \mathrm{c}$ and $3 \mathrm{~d}$. In the case of the preference function calculated with the weight factor $Q_{j}^{a}\left(\omega<8^{\circ}\right)$ (Figure 3c) one can observe the domination of $B$ and $S$ orientations and of orientations lying in the scattering around $S$, or orientations concentrated around the $\{011\}\langle 322\rangle$ position lying in the scattering around $B$. Areas with orientations lying in these regions have on the average between $10-30 \%$ of neighbours with $\omega<8^{\circ}$.

The orientations of areas which have a high number of neighbours with $\omega>20^{\circ}$ as in the case of pure copper, often form tubes lying on the margin of the scattering around the main components $B$ and $S$ (Figure 3d). For these tubes the contribution of neighbours with $\omega>20^{\circ}$ attain values of $70-90 \%$. A rather high contribution of neighbours with $\omega>20^{\circ}$ can also be seen for areas with orientations lying around the $B$ and $G$ positions.

Taking all measuring points together, the number of neighbours in the distance of $\sim 1 \mu \mathrm{m}$ with $\omega>20^{\circ}$ is higher in phosphorus copper than in pure copper. The reverse is true for neighbours with $\omega<8^{\circ}$. The quantitative data are given in Table 1.

For higher values of $|\mathbf{r}|$, namely for $|\mathbf{r}| \cong 2,3$ or $4 \mu \mathrm{m}$, the character of the functions $K_{b}(g)\left(\omega>20^{\circ}\right)$ is in both cases of pure and of phosphorus copper similar to $K_{b}(g)\left(\omega>20^{\circ}\right)$ for $|\mathbf{r}| \cong 1 \mu \mathrm{m}$ (Figure $\left.4 \mathrm{~b}, \mathrm{~d}\right)$. The orientations of areas which have the highest number of more distant neighbours with $\omega>20^{\circ}$ also form tubes lying on the margin of the skeleton line of the main components. However, the contribution of neighbours with $\omega>20^{\circ}$ in the average area is clearly higher in phosphorus copper-than in pure copper (Table 1). 
Table 1 Mean fraction $(\bar{Q})$ of neighbours with small and large disorientation angles $\left(\omega<8^{\circ}\right.$ resp. $\left.\omega>20^{\circ}\right)$ at different distances $|\mathbf{r}|$.

\begin{tabular}{|c|c|c|c|c|c|}
\hline & \multicolumn{4}{|c|}{ Distance $|\boldsymbol{r}|$ in $\mu \mathrm{m}$} \\
\hline & & 1 & 2 & 3 & 4 \\
\hline $\mathrm{Cu}$ & $\omega<8^{\circ}$ & $43 \%$ & $32 \%$ & $28 \%$ & $23 \%$ \\
\hline & $\omega<20^{\circ}$ & $34 \%$ & $39 \%$ & $42 \%$ & $44 \%$ \\
\hline $\mathrm{Cu}+1 \% \mathrm{P}$ & $\omega<8^{\circ}$ & $17 \%$ & $10 \%$ & $7 \%$ & $6 \%$ \\
\hline $\mathrm{Cu}+1 \% \mathrm{P}$ & $\omega>20^{\circ}$ & $51 \%$ & $58 \%$ & $63 \%$ & $66 \%$ \\
\hline
\end{tabular}

In the preference function $K_{a}(g)\left(\omega<8^{\circ}\right.$ and $\left.|\mathbf{r}| \cong 3 \mu \mathrm{m}\right)$ calculated for pure copper (Figure 4a) the $S$ component and some scattering about $S$ dominate. This means that regions where $S$-oriented areas dominate are greater than $C$ or $B$-oriented regions. In contrast, for phosphorus copper the character of the function $K_{a}(g)\left(\omega<8^{\circ}\right.$ and $\left.|\mathbf{r}| \cong 3 \mu \mathrm{m}\right)$ is similar to the function $K_{a}(g)$ calculated for $|\mathbf{r}| \cong 1 \mu \mathrm{m}$.

Partial ODFs selected with respect to special orientations. The preference functions $h_{S}(g), h_{B}(g), h_{C}(g)$ for pure and $h_{B}(g), h_{S}(g), h_{G}(g)$ for phosphorus copper of areas lying in the close $(|\mathbf{r}| \cong 1 \mu \mathrm{m})$ neighbourhood of $S, B, C$ or $G$ oriented crystallites has been calculated. Here the weight factors were set $Q_{i}=1$ for points $P_{i}$ in the nearest neighbourhood of orientations $g$ situated inside a sphere with a radius less than $12^{\circ}$ around the $S, B, C$ or $G$ orientations, respectively. Thus the functions $h(g)$ specify how much more frequently an orientation $g$ occurs in the close neighbourhood of orientation $S, B, C$ or $G$ compared to the texture.

To demonstrate the character of these distributions Figure 5 shows appropriate sections of the preference functions $h_{S}(g), h_{B}(g), h_{C}(g)$ and $h_{G}(g)$ for pure copper and phorphorus copper. In the whole distributions some parallel running tubes can be identified, lying in the scattering around the orientation $S, B, C$ or $G$, respectively.

\section{Partial ODDFs}

The most interesting partial ODDFs from the viewpoint of applications are the general and special preference functions MODFs $m(\Gamma)$ and $m_{g_{0}}(\Gamma)$, which respectively characterize the distribution of the orientation differences between the orientations $g_{i}$ of the various points $P_{i}$ and their close neighbours $P_{i}^{\prime}$, or between the special orientations $g_{0}$ and their nearest neighbours. Only these pairs are given the weight factors $T_{j}=1$ and all others $T_{j}=0$. The resulting partial ODDFs will again be presented by using the axis-angle space.

Pure copper. The MODF $m(\Gamma)=M(\Gamma) / u(\Gamma)$ shown in Figure 6a reveals correlations characterized by maxima at $\sim 60^{\circ}\langle 111\rangle, 35-45^{\circ}\langle 100\rangle$ and at $50-55^{\circ}\langle 110\rangle$. The values at these maxima of about $4.5,5$ or 3.2 indicate how much more frequently these disorientations occur in the close neighbourhood than in $u(\Gamma)$ 


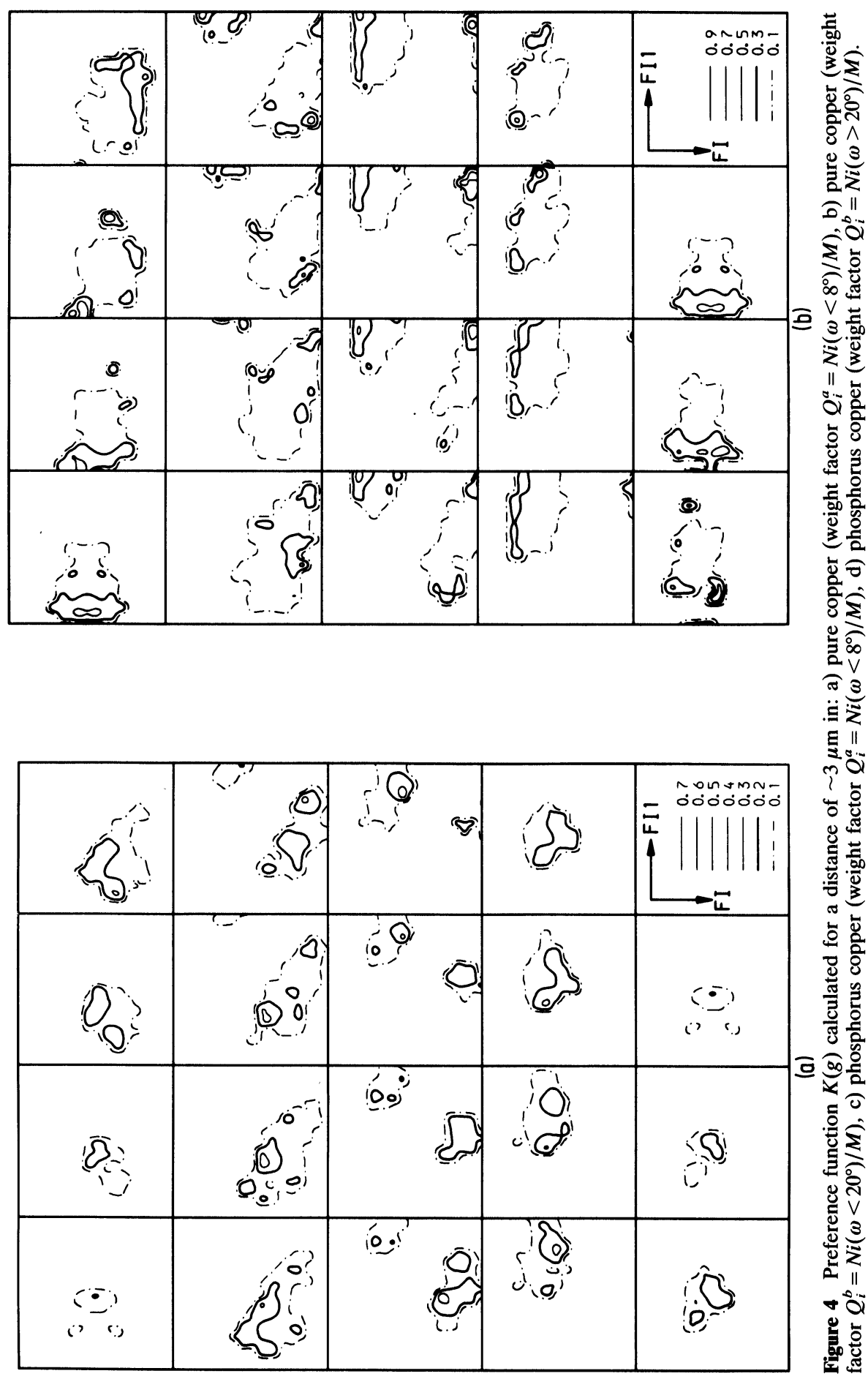


ORIENTATION CHARACTERISTICS

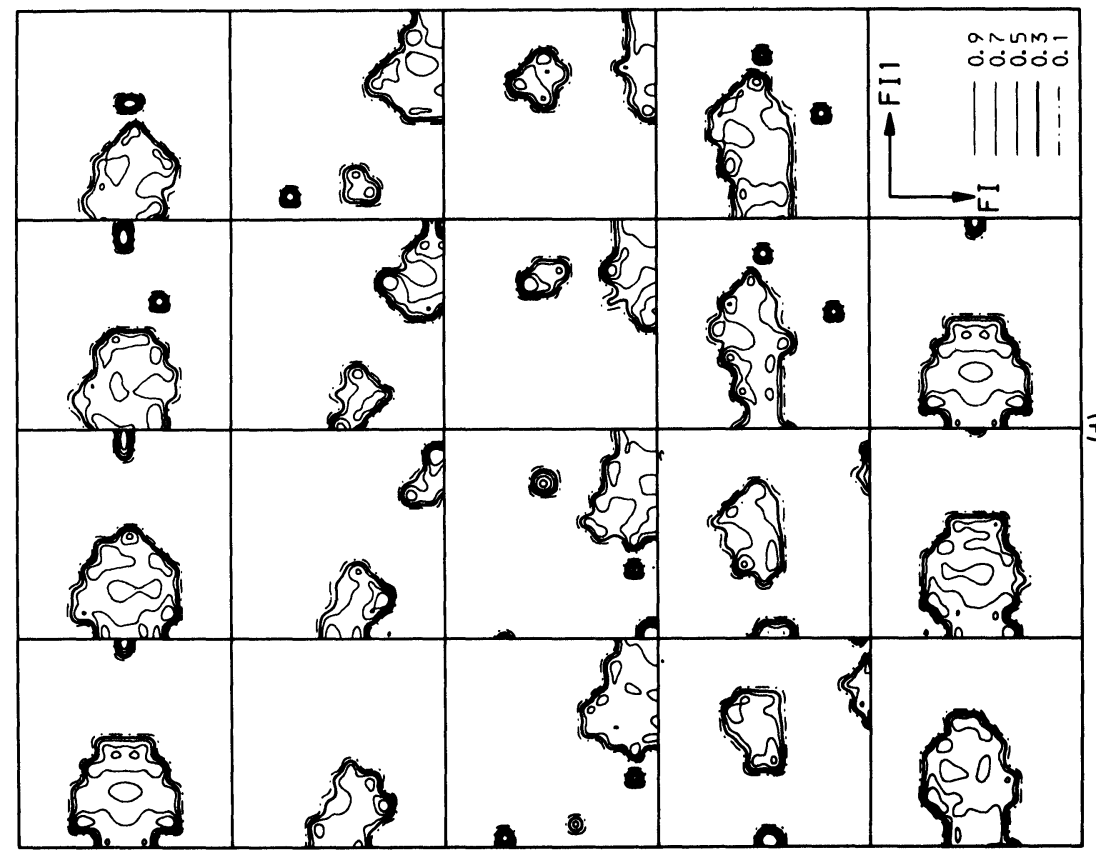

营

这

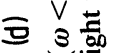

之

ปั

용

要

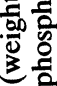

可

这

焉

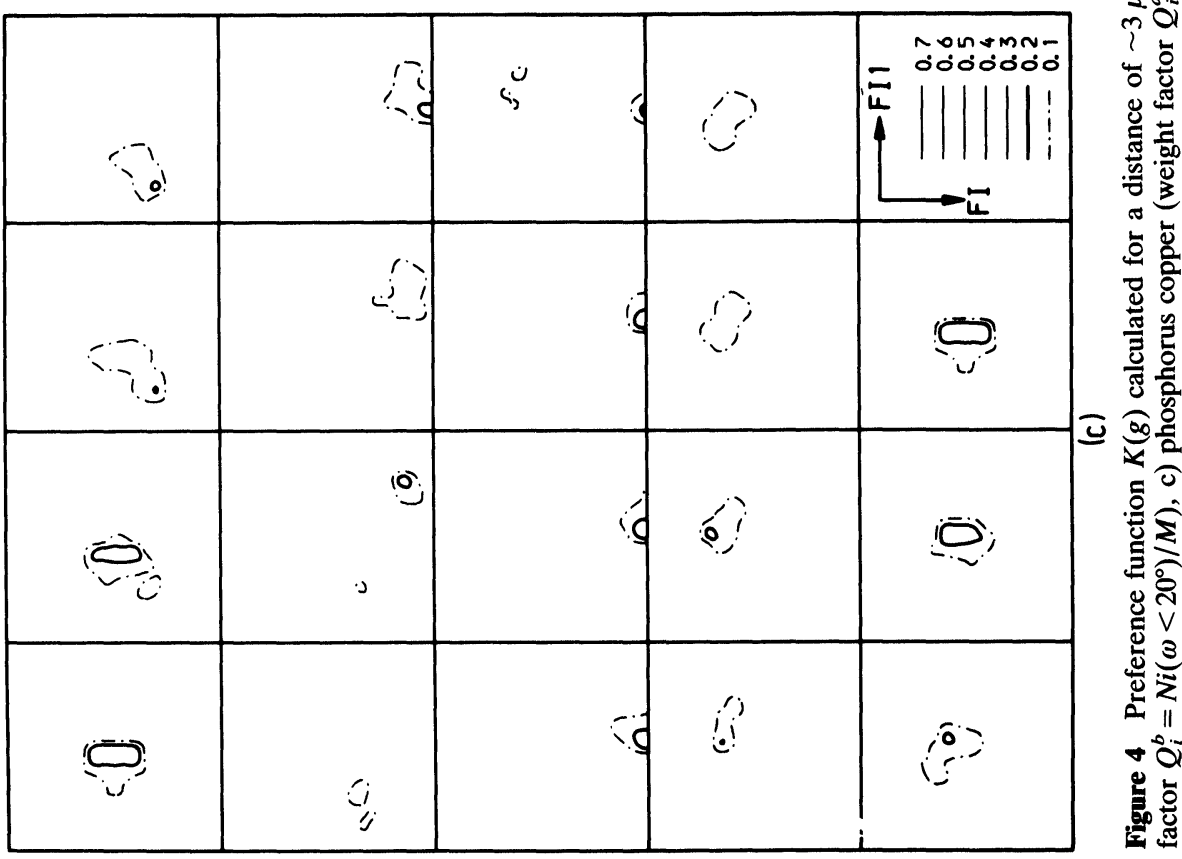




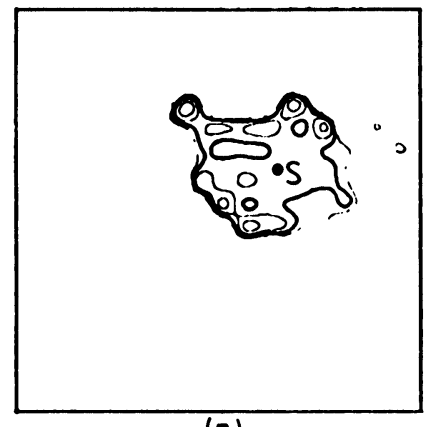

(a)

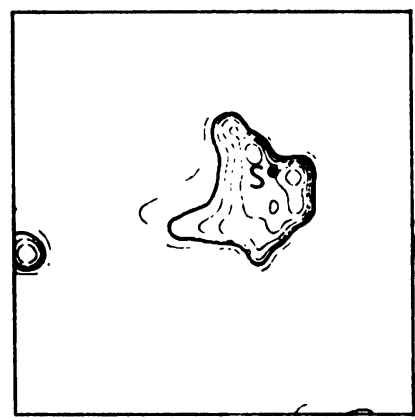

(d)

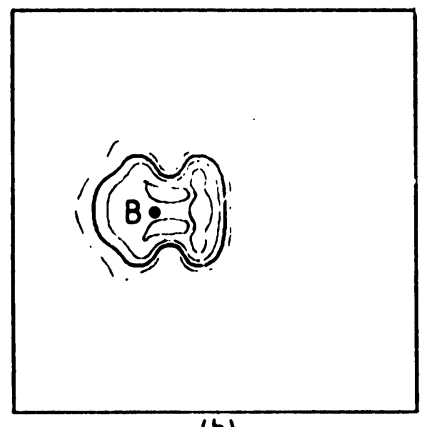

(b)

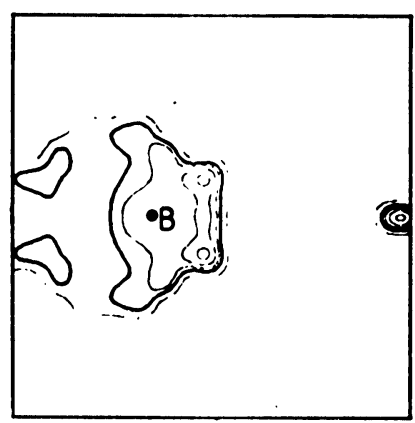

(e)

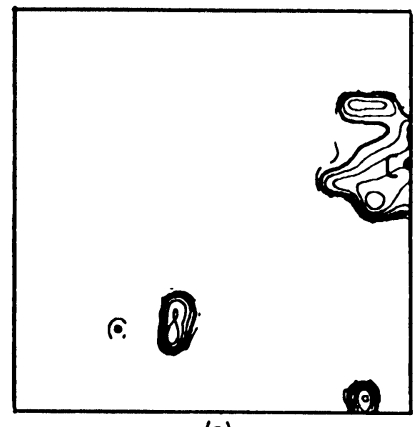

(c)

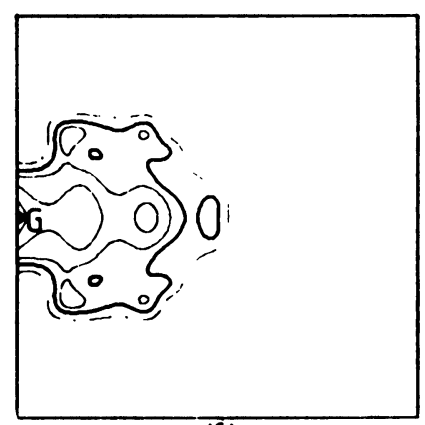

(f)

Figure 5 Preference function $h(g)$ describing the nearest neighbourhood $(|\mathbf{r}| \cong 1 \mu \mathrm{m})$ of the: a) $S$-oriented areas in pure copper $\left(\phi_{2}=65^{\circ}\right.$ section plot of $h(g), 494$ orientations), levels at $1,1.1,1.2$, $\ldots$, b) $B$-oriented areas in pure copper $\left(\phi_{2}=0^{\circ}\right.$ section plot of $h(g), 140$ orientations), levels at 1.0, $2,3, \ldots$, c) $C$-oriented areas in pure copper $\left(\phi=45^{\circ}\right.$ section plot of $h(g), 300$ orientations), levels at $1,1.2,1.4, \ldots$ d) $S$-oriented areas in phosphorus copper $\left(\phi_{2}=65^{\circ}\right.$ section plot of $h(g), 178$ orientations), levels at $0.5,1,1.3,1.6,1.9, \ldots$, e) $B$-oriented areas in phosphorus copper $\left(\phi_{2}=0^{\circ}\right.$ section plot of $h(g), 240$ orientations), levels at $0.5,1.3,1.6,1.9, \ldots$, f) $G$-oriented areas in phosphorus copper $\left(\phi_{2}=0^{\circ}\right.$ section plot of $h(g), 151$ orientations), levels at $0.5,1,1.3,1.6,1.9, \ldots$
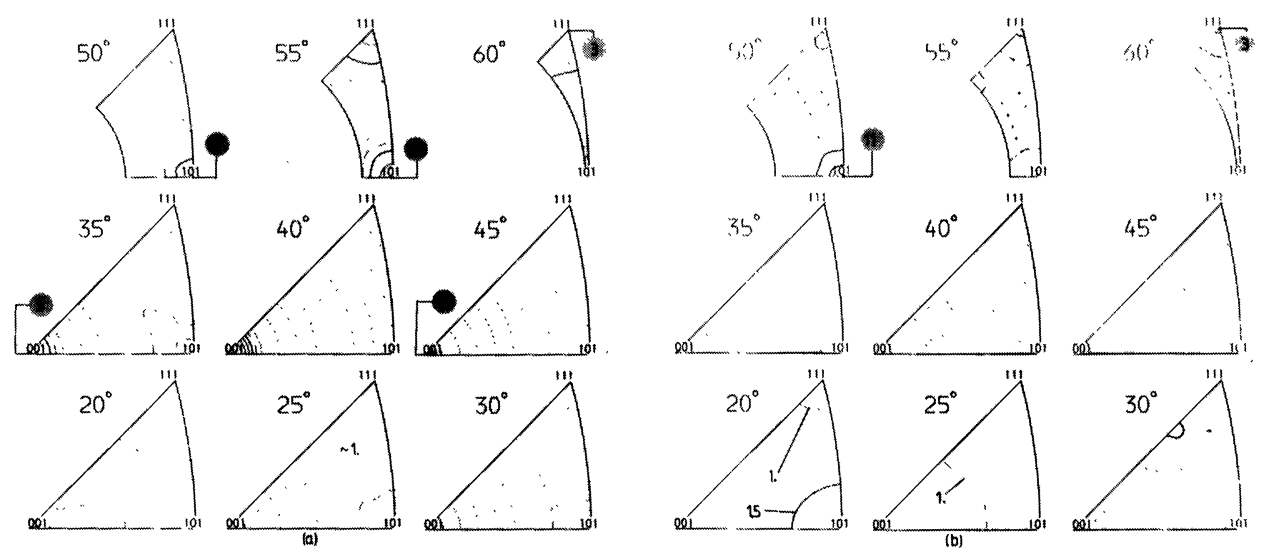

Figure 6 Preference misorientation distribution function MODF $(m(\Gamma)=M(\Gamma) / u(\Gamma),|\mathbf{r}| \cong 1 \mu \mathrm{m}$, $\omega>12^{\circ}$ ) for: a) pure copper, 796 misorientations, levels at $1,2,3, \ldots$, b) phosphorus copper, 838 misorientations, levels at $1,1.5,2, \ldots$ CSL positions are marked with the $\Sigma$ values. 
which shows the distribution of all possible orientation differences in the sample. The character of the distribution of axes of rotation for intermediate values of $\omega$ $\left(10^{\circ}<\omega<20^{\circ}\right)$ is similar to the axes distribution in $u(\Gamma)$. However, the frequency of this type of orientation differences in the nearest neighbourhood of crystallites $(|\mathbf{r}| \cong 1 \mu \mathrm{m})$ is about 2 times higher than in the distribution $u(\Gamma))$. A similar behaviour is observed for small values of $\omega\left(\omega<10^{\circ}\right)$ but here the frequency of disorientations is about 12 times higher than in $u(\Gamma)$. For more distant neighbours i.e. for $|\mathbf{r}| \cong 2,3$ or $4 \mu \mathrm{m}$ the correlations in the MODF disappear slowly. The $60^{\circ}\langle 111\rangle$ relation is still at a distance of $4 \mu \mathrm{m}$ about 3 times higher than in $u(\Gamma)$. Other correlations already disappear at a distance between 2 and $3 \mu \mathrm{m}$.

Finally, the normalized special preference functions MODF $m_{g_{0}}(\Gamma)=$ $M_{g_{0}}(\Gamma) / M(\Gamma)$ with the orientations $g_{0}=S, C$ and $B$ were calculated. These functions indicate how far the close neighbourhood around the special orientations $g_{0}$ is different from the average close neighbourhood. In addition, the neighbourhood of $S, C$ or $B$-oriented crystallinites were divided into two groups: a) neighbours of which the orientations belong to the same group (i.e. $S$-oriented neighbours around $S$-oriented crystallites), b) neighbours of which the orientations do not belong to the same grup (i.e. not $S$-oriented neighbours around $S$-oriented crystallites). Obviously, maxima of the $m_{g_{0}}(\Gamma)$ in case (a) correspond to relations between different complementary components of $S$ orientation, $C, B$ or $G$-orientations.

(i) Neighbourhood of $S$-oriented crystallites. Figures $7 \mathrm{a}$, b show the functions $m_{S S}(\Gamma)$ and $m_{S n s}(\Gamma)$ for $S$-oriented and for not $S$-oriented neighbours respectively. Between the complementary components of $S$-orientation three relations are possible: $\sim 38^{\circ}\langle 111\rangle, \sim 39^{\circ}\langle 221\rangle$ and $\sim 50^{\circ}\langle 332\rangle$. From these three possibilities only the $\sim 40^{\circ}\langle 111\rangle$ relation shows a high maximum $(\sim 6$ times higher than in $M(\Gamma)$ Figure $6 a)$. Instead $50^{\circ}\langle 332\rangle, \sim 60^{\circ}\langle 332\rangle$ relation is observed. The relationships between $S$-oriented crystallites and their not $S$-oriented neighbours are more complicated. Here one can recognize the following maxima: 30$35^{\circ} \sim\langle 210\rangle, \sim 30^{\circ}\langle 111\rangle, \sim 40^{\circ}\langle 110\rangle, 50-55^{\circ}\langle 211\rangle$ and $40^{\circ}\langle 311\rangle$.

(ii) Neighbourhood of $C$-oriented crystallites. Figures $8 \mathrm{a}$, b show the $m_{C C}(\Gamma)$ and $m_{C n C}(\Gamma)$ functions for $C$-oriented and for not $C$-oriented neighbours. Between the complementary components of $C$ orientation only one rotation is possible: $60^{\circ}\langle 111\rangle$. One can recognize this relationship clearly in Figure $8 \mathrm{a}$, here it is about 9 times higher than in the $M(\Gamma)$ distribution. Another maximum observed in this distribution lies in the $50-53^{\circ}\langle 332\rangle$ position. In the $m_{C n C}(\Gamma)$ function (Figure $8 \mathrm{~b}$ ) one can distinguish the following maxima: $\sim 30^{\circ}\langle 110\rangle$, $\sim 50^{\circ}\langle 322\rangle, \sim 55^{\circ}\langle 221\rangle$ and $\sim 50^{\circ}\langle 110\rangle$.

(iii) Neighbourhood of $B$-oriented crystallites. Figures $9 \mathrm{a}$, b show the $m_{B B}(\Gamma)$ and $m_{B n B}(\Gamma)$ functions for $B$-oriented and for not $B$-oriented neighbours. Between the complementary components of $B$ orientation only one rotation is possible: $60^{\circ}\langle 111\rangle$. This rotation is about 6 times more probable than in the case of the average close neighbourhood i.e. in the $M(\Gamma)$ distribution. The $m_{B n B}(\Gamma)$ distribution (Figure $9 \mathrm{~b}$ ) can be characterized by the following rotations: 25$30^{\circ}\langle 110\rangle, 40^{\circ} \sim\langle 311\rangle, 50^{\circ} \sim\langle 322\rangle$ and $60^{\circ} \sim\langle 221\rangle$.

Phosphorus copper. The MODF $m(\Gamma)=M(\Gamma) / u(\Gamma)$ calculated for $|\mathbf{r}| \cong 1 \mu \mathrm{m}$ (Figure 6b) reveals weak correlations characterized by maxima at $60^{\circ}\langle 111\rangle$ and at 

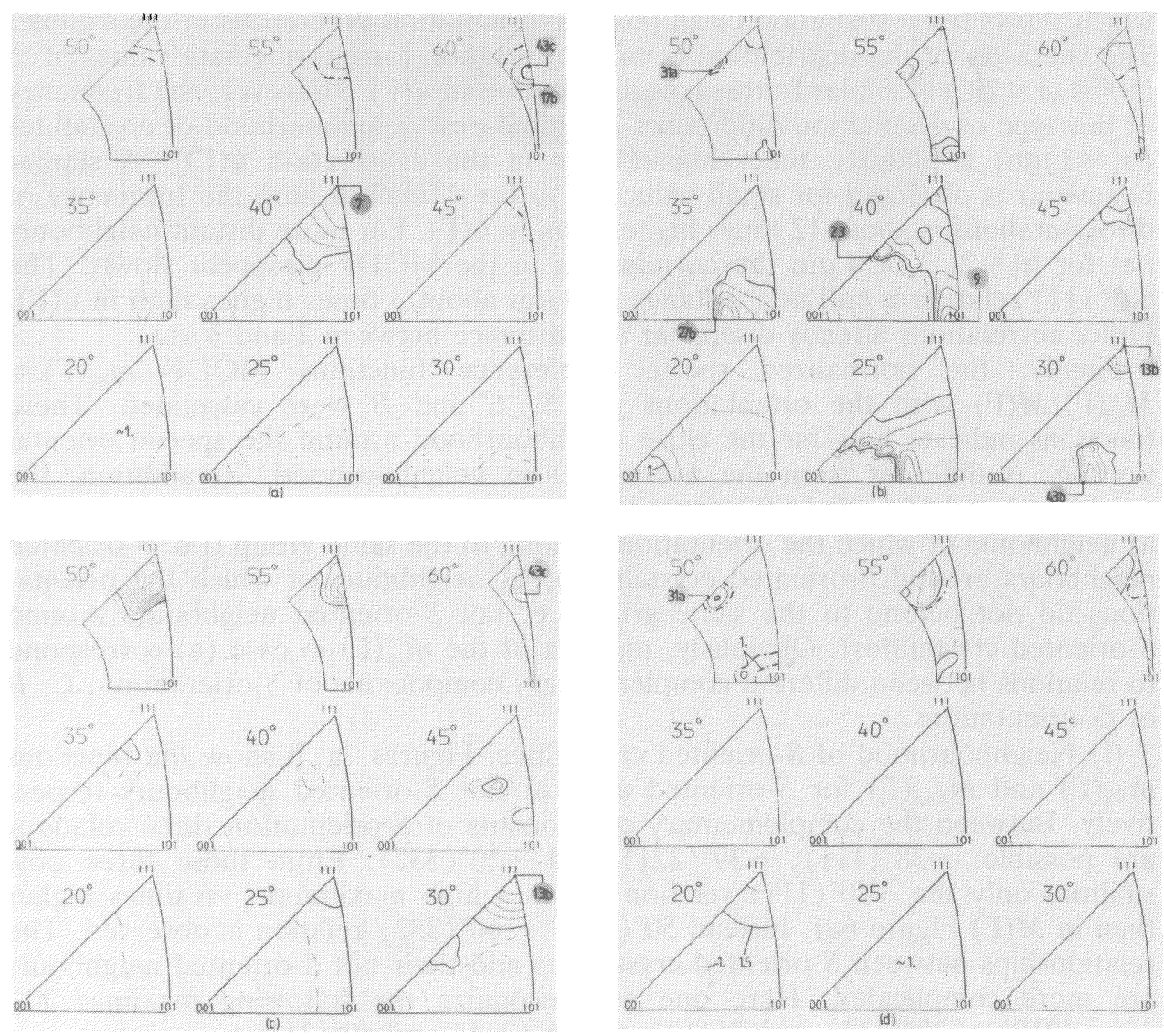

Figure 7 Preference misorientation distribution function $m_{g g^{\prime}}(\Gamma)=M_{g g^{\prime}}(\Gamma) / M(\Gamma), \quad \omega>12^{\circ}:$ a) $m_{S S}(\Gamma), 50$ misorientations between the $S$-oriented areas and their $S$-oriented neighbourhood in pure copper, levels at $1,2,3, \ldots$, b) $m_{S n S}(\Gamma), 356$ misorientations between the $S$-oriented areas and their not $S$-oriented neighbourhood in pure copper, levels at $0.5,1,1.2,1.4,1.6, \ldots, c) m_{s s}(\Gamma), 26$ misorientations between the $S$-oriented areas and their $S$-oriented neighbourhood in phosphorus copper, levels at $1,2,3, \ldots$, d) $m_{S n S}(\Gamma), 202$ misorientations between the $S$-oriented areas and their not $S$-oriented neighbourhood in phosphorus copper, levels at $1,1.5,2.5, \ldots$, CSL positions are marked with the $\Sigma$ values.

$\sim 50^{\circ}\langle 110\rangle$. The disorientations $\sim 60^{\circ}\langle 111\rangle$ in the close neighbourhood of measuring points occur about 1.5 times more frequently and the disorientations $\sim 50^{\circ}\langle 110\rangle$ about 2.1 times more frequently than in the $u(\Gamma)$ calculated for all possible orientation differences in the sample. Up to $\omega=25^{\circ}$ the axes of rotation show no preferred orientations, they are more or less randomly distributed. For $\omega=30^{\circ}$ a weak preference of axes lying in the band between $\langle 111\rangle$ and $\langle 110\rangle$ directions can be observed. For more distant neighbours $(|\mathbf{r}| \cong 2,3$ or $4 \mu \mathrm{m})$ the correlations in the MODF disappear faster than in the case of pure copper. Practically even at $|\mathbf{r}| \cong 2 \mu \mathrm{m} M(\Gamma)$ is the same as $u(\Gamma)$, only the $50^{\circ}\langle 110\rangle$ relation is weakly favoured. 

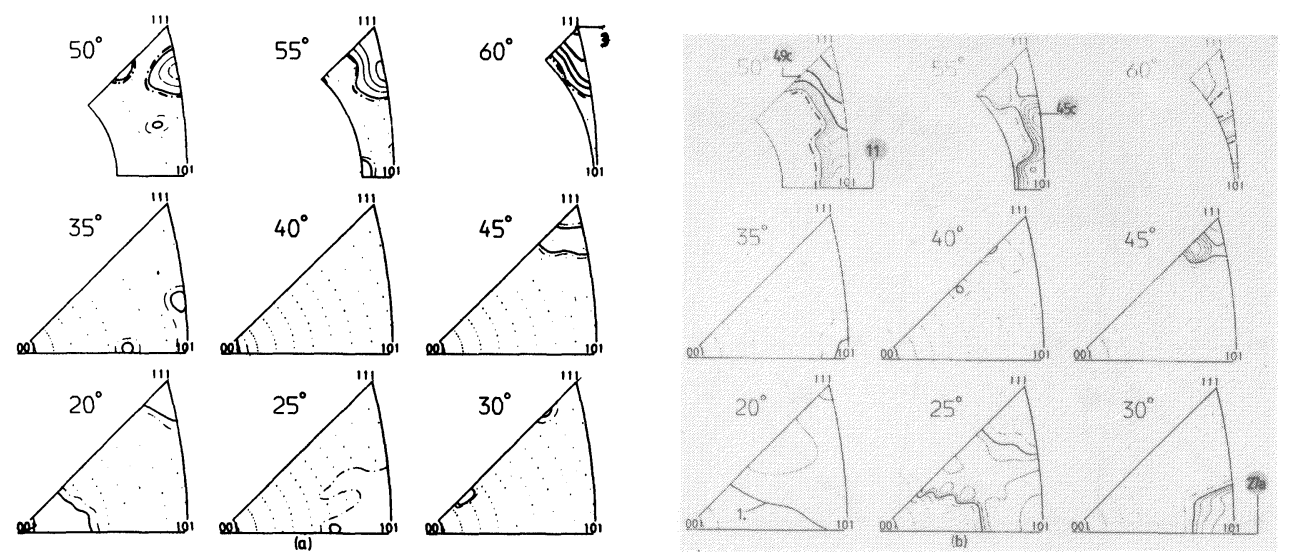

Figure 8 Preference misorientation distribution function $m_{g g^{\prime}}(\Gamma)=M_{g g^{\prime}}(\Gamma) / M(\Gamma), \quad \omega>12^{\circ}:$ a) $m_{C C}(\Gamma), 38$ misorientations between the $C$-oriented areas and their $C$-oriented neighbourhood in pure copper, levels at $0.5,1,3,5, \ldots$, b) $m_{C n C}(\Gamma), 254$ misorientations between the $C$-oriented areas and their not $C$-oriented neighbourhood in pure copper, levels at $0.5,1,1.2,1.4,1.6, \ldots$, CSL positions are marked with the $\Sigma$ values.

As in the case of pure copper, the normalized special preference functions MODF $m_{g_{0}}(\Gamma)=M_{g_{0}}(\Gamma) / M(\Gamma)$ with the orientations $g_{0}=B, S$ and $G$ were calculated. The neighbourhoods of $B, S$ or $G$-oriented crystallites were divided into two groups: a) neighbours of which the orientations belong to the same group b) neighbours of which the orientations do not belong to the same group.

(i) Neighbourhood of $B$-oriented crystallites. Figures $9 \mathrm{c}, \mathrm{d}$ show the $m_{B B}(\Gamma)$ and $m_{B n B}(\Gamma)$ for $B$-oriented and for not $B$-oriented neighbours. Between the complementary components of $B$ orientation one rotation is possible: $60^{\circ}\langle 111\rangle$. In the analysed case this relation is not found, but shifted to the $50^{\circ}\langle 111\rangle$ position which shows a maximum value of $\sim 7$. (Figure 9c). The $m_{B n B}(\Gamma)$ distribution (Figure 9d) shows many similarities with the general MODF $(M(\Gamma))$ : only a weak preference for $\sim 25^{\circ}\langle 110\rangle, \sim 50^{\circ}\langle 211\rangle$ and $\sim 50\langle 430\rangle$ rotations are observed.

(ii) Neighbourhood of $S$-oriented crystallites. Figures 7c, d show the functions $m_{S S}(\Gamma)$ and $m_{S n S}(\Gamma)$ for $S$-oriented and for not $S$-oriented neighbours, respectively. From the three possible relations between the complementary components of $S$ (i.e. $\sim 38^{\circ}\langle 111\rangle, \sim 39^{\circ}\langle 221\rangle$ and $\sim 50^{\circ}\langle 332\rangle$ ), only the $\sim 50^{\circ}\langle 332\rangle$ relation shows a high maximum $(\sim 10$ times higher as in $M(\Gamma))$. Instead a $40^{\circ}\langle 111\rangle$, and a $\sim 30^{\circ}\langle 111\rangle$ relation is observed. The relations between $S$-oriented crystallites and their not $S$-oriented neighbours (Figure 7d) are similar to those in the MODF: only for $55^{\circ}<\omega<60^{\circ}$ and the $\langle 110\rangle, \sim\langle 322\rangle$ directions and for the $\sim 50^{\circ}\langle 211\rangle$ rotation can some preference be noticed.

(iii) Neighbourhood of $G$-oriented crystallites. The angle of rotation between complementary components of $G$ orientation is zero. Thus the $m_{G G}(\Gamma)$ function was not analysed. Figure 10 shows the $m_{G n G}(\Gamma)$ function for not $G$-oriented neighbours. Here the relations $\sim 35^{\circ}\langle 211\rangle, 30-35^{\circ}\langle 110\rangle, 45-50^{\circ}\langle 110\rangle$, and $25-30^{\circ}\langle 100\rangle$ are preferred.

If we consider all the results we obtained on the disorientations, we find that more than $90 \%$ of the disorientations with $\omega>12^{\circ}$ are less than $8^{\circ}$ away from one of the Coincidence-Site Lattices (CSL) orientation relationships $(\Sigma \leq 49)$ (Grimer 

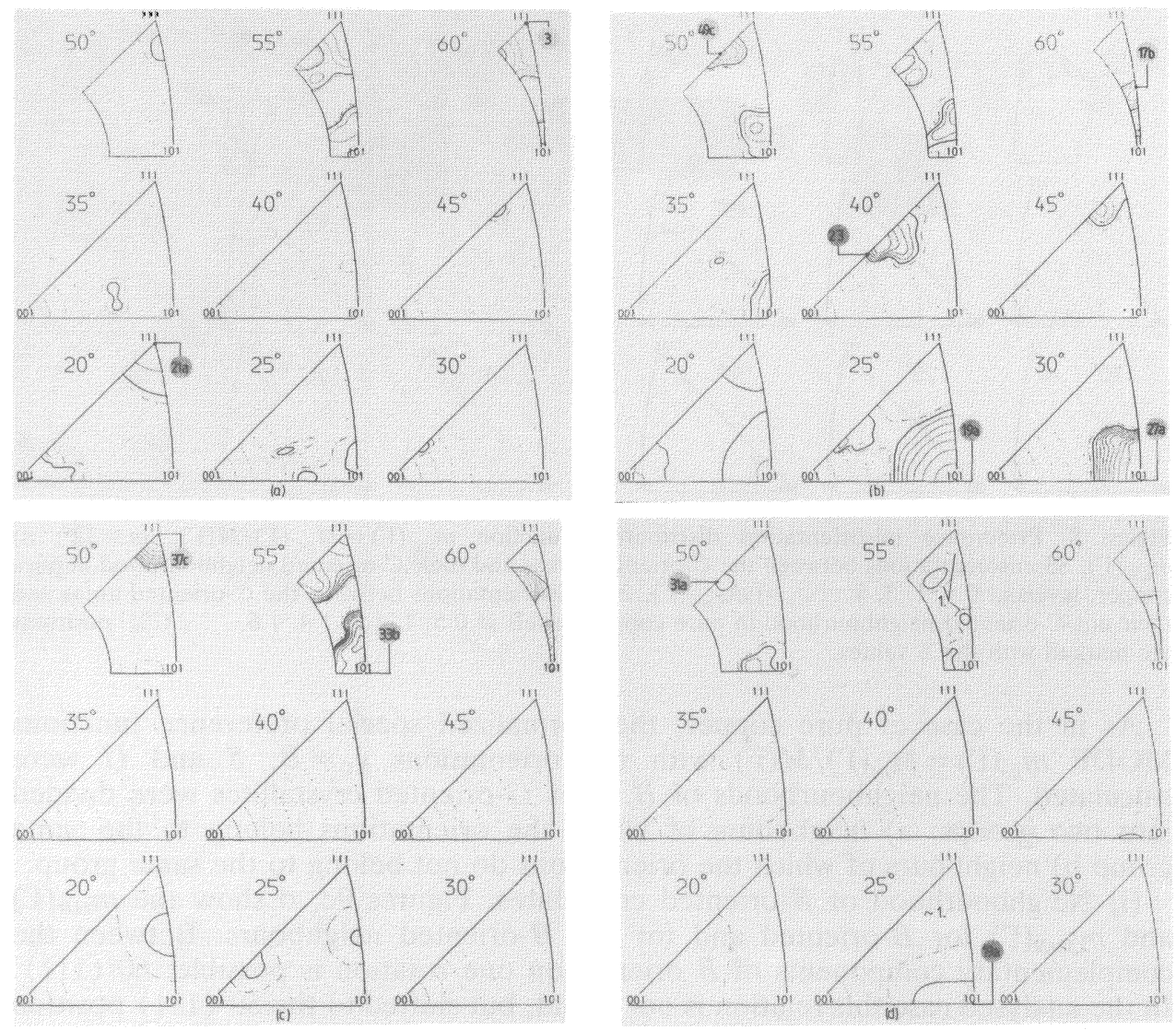

Figure 9 Preference misorientation distribution function $\left.m_{g g^{\prime}}(\Gamma)=M_{g g^{\prime}}(\Gamma) / M(\Gamma), \quad \omega>12^{\circ}: a\right)$ $m_{B B}(\Gamma), 12$ misorientations between the $B$-oriented areas and their $B$-oriented neighbourhood in pure copper, levels at $0.5,1,3,5, \ldots$ b) $m_{B n B}(\Gamma), 73$ misorientation between the $B$-oriented areas and their not $B$-oriented neighbourhood in pure copper, levels at $0.5,1,1.5,2, \ldots, c) m_{B B}(\Gamma), 25$ misorientations between the $B$-oriented areas and their $B$-oriented neighbourhood in phosphorus copper, levels at $1,1.5,2, \ldots$, d) $m_{B n B}(\Gamma), 280$ misorientations between the $B$-oriented areas and their not $B$-oriented neighbourhood in phosphorus copper, levels at $1,1.5,2, \ldots$ CSL positions are marked with the $\Sigma$ values.

et al., 1974). The CLS orientation relationships up to $\Sigma=49$ are shown in detail in Table 2 together with the contributions we found for the close neighbourhood $(|\mathbf{r}|=1 \mu \mathrm{m})$ for the two materials (column "a"). For comparison the probability of the different contributions calculated from the ODF are also listed (column "b"). To assign a particular orientation relationship to a CSL category in Table 2, as in the studies of Warrington and Boon (1975) or Randle et al. (1988), the Brandon's criterion (Brandon, 1966) was used: $\Delta \Omega_{\max }=15^{\circ} \Sigma^{-1 / 2}$, where $\Delta \Omega_{\max }$ is the maximum angular value of the deviation from the ideal CSL disorientation. $\uparrow$ This criterion is fufilled for about $21 \%$ of the disorientations with

$\dagger$ A measured orientation relationship was assigned to the $\Sigma=11$ category for instance, if the deviation from the exact $50.47^{\circ}\langle 110\rangle$ relationship was not larger than $4.5^{\circ}$. 


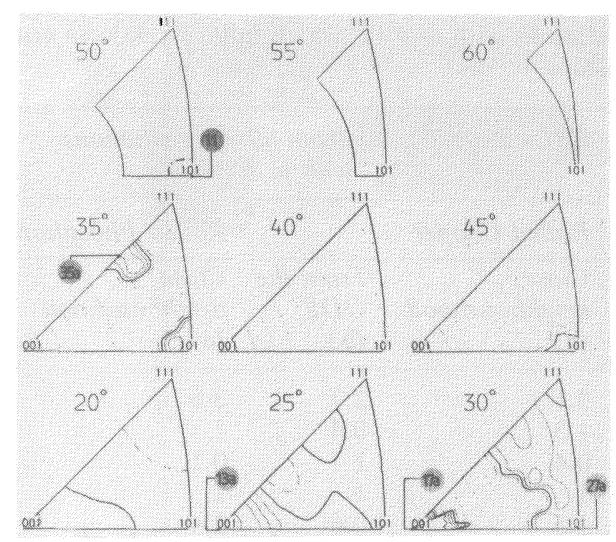

Figure 10 Preference misorientation distribution function $m_{G n G}(\Gamma)=M_{G n G}(\Gamma) / M(\Gamma)$ of 163 misorientations between the $G$-oriented areas and their not $G$-oriented neighbourhood in phosphorus copper, $\omega>12^{\circ}$, levels at $1,1.5,2, \ldots$ CSL positions are marked with the $\Sigma$ values.

$\Sigma \leq 49$ in pure copper and for about $17 \%$ in phosphorus copper (sum of column "a").

\section{DISCUSSION}

The most interesting information resulting from the calculated orientation characteristics concerns the nearest neighbourhood relations. The three main results will now be treated nearer.

(i) In pure copper the disorientation angles $\omega$ between nearest neighbour measuring points are rather small, in phosphorus copper distinctly larger. This observation is directly characterized by the values of the $\bar{Q}$ factors (Table 1 ) giving the mean fractions of neighbours with small or large disorientation angles per measuring point. In pure copper the factor $\bar{Q}$ for disorientation angles $\omega<8^{\circ}$ and for $|\mathbf{r}| \cong 1 \mu \mathrm{m}$ attains the value $\sim 0.43$ i.e. $\sim 43 \%$ of grains lying on a ring with a radius of $\sim 1 \mu \mathrm{m}$ are disoriented relative to the centre by less than $8^{\circ}$. In phosphorus copper this factor is only equal to 0.17 whereas for $\omega>20^{\circ}$ it already has at $|\mathbf{r}| \cong 1 \mu \mathrm{m}$ the high value of 0.51 and even increases further with the distance $|\mathbf{r}|$. This means that in pure copper the distance of $1 \mu \mathrm{m}$ between the nearest measuring points on the grid is comparable to or smaller than the average crystallite size, and that in the material fair-sized $(3,4 \mu \mathrm{m}$ and more) uniformly oriented regions exist. The orientations of these regions often lie not far from the $S$ position. In phosphorus copper the uniformly oriented regions are decidedly smaller. The probability of the existence of such regions greater than $2 \mu \mathrm{m}$ is low. Only grains with orientations lying in the close vicinity of the $S$ or $B$ position have a higher number of neighbours with $\omega<8^{\circ}$. In other words, $S$ and $B$ oriented areas are somewhat larger than others. The distance of $1 \mu \mathrm{m}$ between the nearest measuring points on the grid is supposedly greater in phosphorus copper than the average crystallite size. Thus we note that the orientational details vary on a smaller lateral scale in phosphorus copper than in pure copper. This difference 
Table 2 Fraction of disorientations with $\omega>12^{\circ}$ which fulfill Brandon's criterion for Coincidence-SiteLattice orientation relationships.

\begin{tabular}{|c|c|c|c|c|c|c|}
\hline \multicolumn{2}{|c|}{ CSL-category } & \multicolumn{4}{|c|}{$\begin{array}{c}C S L \text {-category in } \% \text { from all disorientations } \\
\text { with } \omega>12^{\circ}\end{array}$} & \multirow{3}{*}{$\begin{array}{l}\text { Brandon's } \\
\text { criterion: } \\
15^{\circ}(\Sigma)^{-1 / 2} \\
{\left[{ }^{\circ}\right]}\end{array}$} \\
\hline \multirow[t]{2}{*}{ angle axis } & \multirow{2}{*}{$\Sigma$-value } & \multicolumn{2}{|l|}{ Rolled Copper } & \multicolumn{2}{|c|}{ Rolled Phosphorus-Copper } & \\
\hline & & $\begin{array}{l}\text { Close } \\
\text { neighbourhood } \\
\text { (a) }\end{array}$ & $\begin{array}{l}\text { From the } \\
O D F \\
\text { (b) }\end{array}$ & $\begin{array}{l}\text { Close } \\
\text { neighbourhood } \\
\text { (a) }\end{array}$ & $\begin{array}{l}\text { From the } \\
\text { ODF } \\
\text { (b) }\end{array}$ & \\
\hline $60^{\circ}\langle 111\rangle$ & 3 & 9.1 & 2.3 & 3.6 & 2.5 & 8.7 \\
\hline $37^{\circ}\langle 100\rangle$ & 5 & 1.3 & 0.4 & 1. & 0.5 & 6.7 \\
\hline $38^{\circ}\langle 111\rangle$ & 7 & 0.4 & 1.7 & 0.1 & 0.8 & 5.7 \\
\hline $39^{\circ}\langle 110\rangle$ & 9 & 1. & 1. & 1. & 0.9 & 5. \\
\hline $50^{\circ}\langle 110\rangle$ & 11 & 1.5 & 0.6 & 2.4 & 0.6 & 4.5 \\
\hline $23^{\circ}\langle 100\rangle$ & $13 a$ & 0.3 & 0.3 & 0.7 & 0.6 & 4.2 \\
\hline $28^{\circ}\langle 111\rangle$ & $13 b$ & 0.6 & 0.6 & 0.5 & 0.5 & 4.2 \\
\hline $48^{\circ}\langle 210\rangle$ & 15 & 0.1 & 0.3 & 0.5 & 0.4 & 3.9 \\
\hline $28^{\circ}\langle 100\rangle$ & $17 \mathrm{a}$ & 0. & 0.1 & 0.3 & 0.2 & 3.6 \\
\hline $62^{\circ}\langle 221\rangle$ & $17 b$ & 0.1 & 0.4 & 0.4 & 0.4 & 3.6 \\
\hline $27^{\circ}\langle 110\rangle$ & $19 a$ & 0.8 & 0.5 & 0.8 & 0.5 & 3.4 \\
\hline $47^{\circ}\langle 111\rangle$ & $19 b$ & 0.1 & 0.3 & 0. & 0.2 & 3.4 \\
\hline $22^{\circ}\langle 211\rangle$ & $21 \mathrm{a}$ & 0.3 & 0.4 & 0.3 & 0.4 & 3.3 \\
\hline $44^{\circ}\langle 211\rangle$ & $21 b$ & 0.3 & 0.5 & 0.3 & 0.4 & 3.3 \\
\hline $40^{\circ}\langle 311\rangle$ & 23 & 0.3 & 0.3 & 0.1 & 0.2 & 3.1 \\
\hline $16^{\circ}\langle 100\rangle$ & $25 a$ & 0.4 & 0.2 & 1. & 0.3 & 3. \\
\hline $52^{\circ}\langle 331\rangle$ & $25 \mathrm{~b}$ & 0.3 & 0.5 & 0.5 & 0.5 & 3. \\
\hline $32^{\circ}\langle 110\rangle$ & $27 a$ & 0.1 & 0.2 & 0.5 & 0.2 & 2.9 \\
\hline $35^{\circ}\langle 210\rangle$ & $27 b$ & 0.3 & 0.3 & 0.3 & 0.3 & 2.9 \\
\hline $44^{\circ}\langle 100\rangle$ & $29 a$ & 0.1 & 0.01 & 0. & 0.02 & 2.8 \\
\hline $46^{\circ}\langle 221\rangle$ & $29 b$ & 0.4 & 0.3 & 0.1 & 0.3 & 2.8 \\
\hline $52^{\circ}\langle 211\rangle$ & $31 \mathrm{a}$ & 0.3 & 0.3 & 0. & 0.2 & 2.7 \\
\hline $18^{\circ}\langle 111\rangle$ & $31 \mathrm{~b}$ & 0.3 & 0.3 & 0.3 & 0.3 & 2.7 \\
\hline $34^{\circ}\langle 311\rangle$ & $33 a$ & 0.1 & 0.2 & 0. & 0.2 & 2.6 \\
\hline $57^{\circ}\langle 110\rangle$ & $33 b$ & 0.7 & 0.1 & 0. & 0.2 & 2.6 \\
\hline $34^{\circ}\langle 211\rangle$ & $35 a$ & 0. & 0.3 & 0.3 & 0.2 & 2.5 \\
\hline $43^{\circ}\langle 331\rangle$ & $35 \mathrm{~b}$ & 0.1 & 0.2 & 0.4 & 0.2 & 2.5 \\
\hline $19^{\circ}\langle 100\rangle$ & $37 a$ & 0. & 0.01 & 0. & 0.01 & 2.5 \\
\hline $43^{\circ}\langle 310\rangle$ & $37 b$ & 0.1 & 0.1 & 0. & 0.1 & 2.5 \\
\hline $51^{\circ}\langle 111\rangle$ & $37 c$ & 0. & 0.04 & 0. & 0.05 & 2.5 \\
\hline $32^{\circ}\langle 111\rangle$ & $39 a$ & 0. & 0.05 & 0. & 0.02 & 2.4 \\
\hline $50^{\circ}\langle 321\rangle$ & $39 b$ & 0.1 & 0.4 & 0. & 0.3 & 2.4 \\
\hline $18^{\circ}\langle 100\rangle$ & $41 a$ & 0. & 0. & 0. & 0. & 2.3 \\
\hline $41^{\circ}\langle 210\rangle$ & $41 b$ & 0. & 0.1 & 0. & 0.1 & 2.3 \\
\hline $56^{\circ}\langle 110\rangle$ & $41 c$ & 0.1 & 0.01 & 0. & 0.01 & 2.3 \\
\hline $15^{\circ}\langle 111\rangle$ & $43 a$ & 0. & 0.1 & 0. & 0.1 & 2.3 \\
\hline $28^{\circ}\langle 210\rangle$ & $43 b$ & 0.1 & 0.2 & 0.3 & 0.3 & 2.3 \\
\hline $61^{\circ}\langle 332\rangle$ & $43 c$ & 0.1 & 0.1 & 0. & 0.1 & 2.3 \\
\hline $29^{\circ}\langle 311\rangle$ & $45 a$ & 0.3 & 0.2 & 0.5 & 0.2 & 2.2 \\
\hline $37^{\circ}\langle 221\rangle$ & $45 b$ & 0. & 0.2 & 0.1 & 0.1 & 2.2 \\
\hline $53^{\circ}\langle 221\rangle$ & $45 c$ & 0.3 & 0.2 & 0.3 & 0.2 & 2.2 \\
\hline $37^{\circ}\langle 331\rangle$ & $47 a$ & 0.3 & 0.2 & 0.4 & 0.1 & 2.2 \\
\hline $44^{\circ}\langle 320\rangle$ & $47 b$ & 0. & 0.1 & 0. & 0.1 & 2.2 \\
\hline $44^{\circ}\langle 111\rangle$ & $49 a$ & 0. & 0. & 0. & 0. & 2.1 \\
\hline $44^{\circ}\langle 511\rangle$ & $49 b$ & 0.1 & 0.04 & 0. & 0.05 & 2.1 \\
\hline $49^{\circ}\langle 322\rangle$ & $49 c$ & 0.3 & 0.2 & 0. & 0.2 & 2.1 \\
\hline
\end{tabular}

a: Disorientations determined between nearest neighbours.

b: Probability of the disorientations calculated from the ODF. 
between a material with a copper and brass-type rolling texture has already been suggested earlier, based on Laue diffraction patterns (Haeßner and Keil, 1967).

(ii) It is immediately obvious from Figure 6a that in deformed copper there must exist a larger number of twin (or near twin) boundaies than would be expected from calculations based on the assumption of a random neighbourhood of grains (compare the ODDF Figure 2a and MDF Figure 6a). The same difference is also recognized from Table 2 (compare column " $a$ " and " $b$ " for $\left.60^{\circ}\langle 111\rangle\right)$. The nearest neighbour pairs belonging to the twin relation $60^{\circ}\langle 111\rangle$ are very often of the $\mathrm{C}-\mathrm{C}$ or B-B type, i..e they are pairs where one point in the material is $C$ or $B$ oriented and the second point belongs to another complementary variant of the $C$ or $B$ orientation respectively. Most of them are a few degrees away from the $60^{\circ}\langle 111\rangle$ relationship. They are probably of the incoherent type which can be concluded from the associated bright images. Coherent twin boundaries were not observed. These structural details are significant in the description of models on the origin of annealing twins (Nielsen, 1966; Kurzydlowski, 1991). Besides the twin relation $(\Sigma=3)$, two other CSL orientation relationships, namely the $35-40^{\circ}\langle 100\rangle \Sigma=5$ and $50^{\circ}\langle 110\rangle \Sigma=11$ occur decidely more frequently as would be expected from texture calculations.

The increased occurrence of these relationships in rolled copper can be for two quite different reasons.

- The first is that these relationships exist between the different fragments of the deformed matrix. For instance, the twin orientation relationship exists between complementary variants of $C$ or $B$ orientations. This reason is directly related to the ODF of the material.

- The second reason is that specially oriented fragments of the deformed matrix are clustered. This reason is of a topological nature and is related to the nonrandom spatial arrangement of orientations in the sample ("spatial orientation clustering").

The opposite tendency to the behaviour described above can also be observed: the orientation relationship $\sim 40^{\circ}\langle 111\rangle(\Sigma=7)$, which occurs between complementary variants of the $S$ orientation is observed not as frequently as expected from texture calculations (see Table 2). This is related to the fact that $S$-oriented crystallites often form uniformly oriented regions which are decidedly larger than differently oriented regions. This can be called "spatial orientation ordering" in contrast to clustering.

In phosphorus copper the same orientation relationships namely $60^{\circ}\langle 111\rangle$ and $50^{\circ}\langle 110\rangle$ are distinguished. This means that, as in pure copper, there exists the tendency to spatial orientation clustering for these orientations. However, this tendency in the case of $60^{\circ}\langle 111\rangle$ is not as distinct as in pure copper.

(iii) The exact analysis of the close neighbourhood relations (distance $\approx 1 \mu \mathrm{m}$ ) around $S, C, B$, or $G$ oriented crystallites shows some general similarities in both materials, but some interesting differences in detail. Thus the neighbourhood of $S$ oriented crystallites $\left(g_{s}\right)$ for both materials also consists of about $12 \%$ (50 of all 406 pairs in pure copper and 26 of all 228 pairs in phosphorus copper; disorientation angle $\omega>12^{\circ}$ ) of $S$-oriented crystallites. Of these three relationships between complementary variants of the orientation $g_{s}$, which are described by the orientation differences $\Gamma_{r}=g_{s} L_{r}^{2} g_{s}^{-1}\left(L_{r}^{2}\right.$ are rotation elements of the 
sample symmetry), only one is found each time in the special MODF (Figures 7a and $7 \mathrm{c}$ ): in pure copper the $40^{\circ}\langle 111\rangle$ orientation difference or the $50^{\circ}\langle 332\rangle$ in phosphorus copper. $\dagger$ The $39^{\circ}\langle 221\rangle$ variant in pure copper falls into the extended scattering region around the $40^{\circ}\langle 111\rangle$ maximum and is therefore not visible; in phosphorus copper this does not occur. The last of the three variants is shifted to another position: in pure copper the $50^{\circ}\langle 332\rangle$ to the $60^{\circ}\langle 332\rangle$ position, in phosphorus copper the $40^{\circ}\langle 111\rangle$ to the $30^{\circ}\langle 111\rangle$ position.

For the $C$ or $B$ oriented crystallites $\left(g_{C}\right.$ or $\left.g_{B}\right)$ in pure copper the relationships are very similar. About $13 \%$ of the neighbours (38 of all 292 pairs for $C$ and 12 of all 85 pairs for $B$; disorientation angle $\omega>12^{\circ}$ ) are also $C$ or $B$-oriented crystallites. Since here only one complementary relationship $\left(60^{\circ}\langle 111\rangle\right)$ is possible, it is clearly visible in the special MODFs (Figures $8 \mathrm{a}$ and $9 \mathrm{a}$ ). In phosphorus copper the situation for the $B$-oriented crystallites is different from pure copper insofar as on the one hand only $8 \%$ of the neighbours are also $B$-oriented crystallites ( 25 of all 305 pairs; disorientation angle $\omega>12^{\circ}$ ), and on the other hand a maximum is not observed at the $60^{\circ}\langle 111\rangle$ position but rather at $50^{\circ}\langle 111\rangle$.

The neighbourhood majority (for disorientation angle $\omega>12^{\circ}$ ) around $S, C$, or $B$ oriented crystallites is not oriented according to $S, C$ or $B$ in both materials. Generally the partial orientation difference distributions for these neighbours are much more scattered in phosphorus copper than in pure copper. The maxima in phosphor copper are not so exactly defined and thus an identification of special CLS orientation relationships is not so unequivocal as in pure copper. Since the structural details change faster locally in phosphorus copper than in pure copper, these differences in the distribution functions can also be the result of the uniformly equal grid length of $\sim 1 \mu \mathrm{m}$. Orientation measurements in smaller distances than $1 \mu \mathrm{m}$ should therefore clarify this.

Finally, we may point out that in practically every case the orientation parameters determined from the partial MODF's can be easily assigned to CSL relationships (see Table 2). This good agreement just means in the first instance that the CSL-method of analysis can also be used to describe the neighbourhood relationships in rolled materials. This does not imply a statement about the possible underlying causes.

\section{ACKNOWLEDGEMENT}

We would like to thank the Deutsche Forschungsgemeinschaft for financial support.

\section{References}

Adams, B. L., Morris, P. R., Wang, T. T., Wilden, K. S. and Wright, S. I. (1987). Description of Orientation Coherence in Polycrystalline Materials. Acta Metallurgica, 35, 2935-2946.

Brandon, D. G. (1966). The Structure of High-Angle Grain Boundaries. Acta Metallurgica, 14, 1479-1484.

\footnotetext{
$\dagger$ In sample space $40^{\circ}\langle 111\rangle$ corresponds to a rotation around the sheet normal; and $50^{\circ}\langle 332\rangle$ is a rotation around the rolling direction.
} 
Bunge, H. J. (1982). Textures Analysis in Materials Science. London, Butterworths.

Grimmer, H., Bollman, H. and Warrington, D. H. (1974). Coincidence-Site Lattices and Complete Pattern-Shift Lattices in Cubic Crystals. Acta Crystallographica, A30, 197-207.

Haeßner, F. and Keil, D. (1967). Orientierungsverteilung der Kristallite in gewalztem Messing 70, bestimmt mit Hilfe elektronenmikroskopischer Feinbereichsbeugung. Zeitschrift für Metallkunde, 58, 220-227.

Haeßner, F., Pospiech, J. and Sztwiertnia, K. (1983). Spatial Arrangement of Orientations in Rolled Copper. Materials Science and Engineering, 57, 1-14.

Heilman, P., Clark, W. A. T. and Rigney, D. A. (1982). Computerized Method to Determine Crystal Orientations from Kikuchi Patterns. Ultramicroscopy, 9, 365-372.

Kurzydłowski, K. J. (1991). On the Formation of Twin Grains as a Result of Grain Encounters during the Process of Recrystallization and Grain Growth. Scripta Metallurgica, 25, 1099-1102.

Morawiec, A. and Pospiech, J. (1992). Functions Describing Orientation Correlations in Polycrystalline Materials. Textures and Microstructures 19, 67-74.

Nielsen, J. P. (1967). The Origin of Annealing Twins. Acta Metallurgica, 15, 1083-1085.

Pospiech, J. and Lücke, K. (1975). The Rolling Textures of Copper and $\alpha$-Brasses Discussed in Terms of the Orientation Distribution Function. Acta Metallurgica, 23, 997-1007.

Pospiech, J., Sztwiertnia, K. and Haeßner, F. (1986). The Misorientation Distribution Function. Textures and Microstructures, 6, 201-215.

Pospiech, J., Lücke, K. and Sztwiertnia, K. (1991). Orientation Characteristics of the Microstructure. In Proceedings of the ICOTOM 9 (Avignon 1990, France), ed. H. J. Bunge, special issue of Textures and Microstructures, Gordon and Breach Science Publishers, pp. 103-108.

Pospiech, J., Lücke, K. and Sztwiertnia, K. Orientation Distribution and Orientation Correlation Functions for Description of Microstructures. Submitted to Acta Metallurgica.

Randle, V., Ralph, B. and Dingley, D. (1988). The Relationship between Microstructure and Grain Boundary Parameters. Acta Metallurgica, 36, 267-273.

Shvindlerman, L. S. and Straumal, B. B. (1985). Regions of Existence of Special and non-Special Grain Boundaries. Acta Metallurgica, 33, 1735-1749.

Virnich, K. H. (1979). Orientierungsverteilungen in gewalzten und primär rekristallisierten Blechen aus Kupfer und binären Kupferlegierungen. Doctoral Thesis, RWTH Aachen.

Warrington, D. H. and Boon, M. (1975). Ordered Structures in Random Grain Boundaries. Acta Metallurgica, 23, 599-607.

Weiland, H. and Schwarzer, R. (1986). On-line Texture Determination by Kikuchi or Channeling Patterns. In Experimental Techniques of Texture Analysis, ed. H. J. Bunge, DGM Informationsgesellschaft, Oberursel 1986, 301-313. 